\title{
Naturally Occurring Flavonoids and Isoflavonoids and Their Microbial Transformation: A Review
}

\author{
Jun-Fei Wang ${ }^{1,+}$, Si-Si Liu ${ }^{1,+}$, Zhi-Qiang Song ${ }^{1}$, Tang-Chang Xu ${ }^{1}$, Chuan-Sheng Liu ${ }^{1}$, \\ Ya-Ge Hou ${ }^{1}$, Rong Huang ${ }^{2}$ and Shao-Hua $\mathrm{Wu}^{1, * \mathbb{D}}$ \\ 1 Yunnan Institute of Microbiology, School of Life Sciences, Yunnan University, Kunming 650091, China; \\ wang_junfei@163.com (J.-F.W.); liusisi1994@126.com (S.-S.L.); songzhiqiang1996@126.com (Z.-Q.S.); \\ tcxu123@126.com (T.-C.X.); liucs313@126.com (C.-S.L.); houyage@126.com (Y.-G.H.) \\ 2 School of Chemical Science and Technology, Yunnan University, Kunming 650091, China; \\ huangrong@ynu.edu.cn \\ * Correspondence: shhwu@ynu.edu.cn or shwu123@126.com \\ + These authors contributed equally to this work.
}

Academic Editor: Nawaf Al-Maharik

Received: 14 October 2020; Accepted: 1 November 2020; Published: 3 November 2020

\begin{abstract}
Flavonoids and isoflavonoids are polyphenolic secondary metabolites usually produced by plants adapting to changing ecological environments over a long period of time. Therefore, their biosynthesis pathways are considered as the most distinctive natural product pathway in plants. Seemingly, the flavonoids and isoflavones from fungi and actinomycetes have been relatively overlooked. In this review, we summarized and classified the isoflavones and flavonoids derived from fungi and actinomycetes and described their biological activities. Increasing attention has been paid to bioactive substances derived from microorganism whole-cell biotransformation. Additionally, we described the utilization of isoflavones and flavonoids as substrates by fungi and actinomycetes for biotransformation through hydroxylation, methylation, halogenation, glycosylation, dehydrogenation, cyclisation, and hydrogenation reactions to obtain rare and highly active biofunctional derivatives. Overall, among all microorganisms, actinomycetes are the main producers of flavonoids. In our review, we also summarized the functional genes involved in flavonoid biosynthesis.
\end{abstract}

Keywords: flavonoids; isoflavonoids; fungi; actinomycetes; biotransformation; biosynthesis

\section{Introduction}

Flavonoids and isoflavonoids are versatile natural compounds and subdivisions of polyphenols that represent a large proportion of secondary metabolites produced by higher plants and are a rich part of the human diet [1]. They are playing multiple roles in the physiology and ecology of individual plant species. Flavonoids are a type of yellow pigment derived from 2-phenyl chromogenone as the parent nucleus and a series of compounds with $\mathrm{C} 6-\mathrm{C} 3-\mathrm{C} 6$ as the basic skeleton. Isoflavonoids are a subclass of flavonoids characterized by possessing a benzene-ring connected to C-3 instead of C-2 [2]. Phenylpropanoid and polyketone compounds are normally catalyzed by chalcone synthase to produce chalcones, and then cyclization of chalcones leads to generate flavonoids. Isoflavonoids, originating from the same biochemical pathway as flavonoids, are derived by aryl migration in a 2-phenylchroman skeleton under the catalysis of 2-hydroxyisoflavanone synthase [3-6]. Flavonoids and isoflavonoids are well-known natural products with extensive pharmacological activities and extremely low toxicity and therefore have become the focus and hotspots of drug discovery and development [7-9]. Most plants contain isoflavonoids and flavonoids that play important roles in the growth and development of plants as well as in antibacterial and disease-preventing aspects [10,11]. More importantly, they possess 
a wide range of biological activities, such as antibacterial, antifungal, antiviral [12,13], antitumour [8], anti-inflammatory [14], and antiaging activities [15].

Most research on isoflavonoids and flavonoids has focused on plant sources, and relatively less attention has been paid on microorganisms as the potential sources of these compounds. The presence of metabolic pathways of isoflavonoid and flavonoid biosynthesis in microorganisms has been confirmed. In particular, the presence of isoflavonoids and flavonoids in fungi and actinomycetes has been widely reported in studies $[16,17]$. Both fungi and actinomycetes exhibit unique and unusual biochemical pathways, and many important drugs have been derived from their secondary metabolites, for instance, penicillin, cyclosporin, paclitaxel, and statins are derived from fungi [18], and streptomycin, kanamycin, nystatin, and rifamycin are derived from actinomycetes [19,20]. Therefore, isoflavonoids and flavonoids derived from fungi and actinomycetes are of great significance. Fungi and actinomycetes are also the key biological sources of isoflavonoid and flavonoid biotransformation [21,22]. Microbial transformation is advantageous because of mild reaction conditions and high regio- and enantioselectivity. The literature suggests that almost all isoflavonoids and flavonoids derived from actinomycetes are produced by Streptomyces because the genes in this genus encode key enzymes such as phenylalanine ammonia-lyase and chalcone synthase (CHS) that catalyze isoflavone and flavone synthesis [23,24].

\section{Isoflavonoids and Flavonoids from Actinomycetes and Fungi}

\subsection{Isoflavonoids}

Prior to 2000, plant-derived natural products of isoflavones were mainly confined to the family Leguminosae $[25,26]$. Since then, several comprehensive reviews on the occurrence of isoflavones in non-leguminous plants have been published [27-29]. Recent reviews have focused on newly discovered isoflavonoids in legumes $[30,31]$. However, only a few of these compounds have been isolated from fungi and actinomycetes. Here, we classified the isoflavones isolated from fungi and actinomycetes into three categories, namely simple isoflavones, isoflavone glycosides, and complex isoflavones; the structures of the compounds are shown in Figures 1-3, respectively. These compounds mainly exhibit antioxidant, anti-tumour, antimicrobial, and $\beta$-galactosidase inhibitory activities.

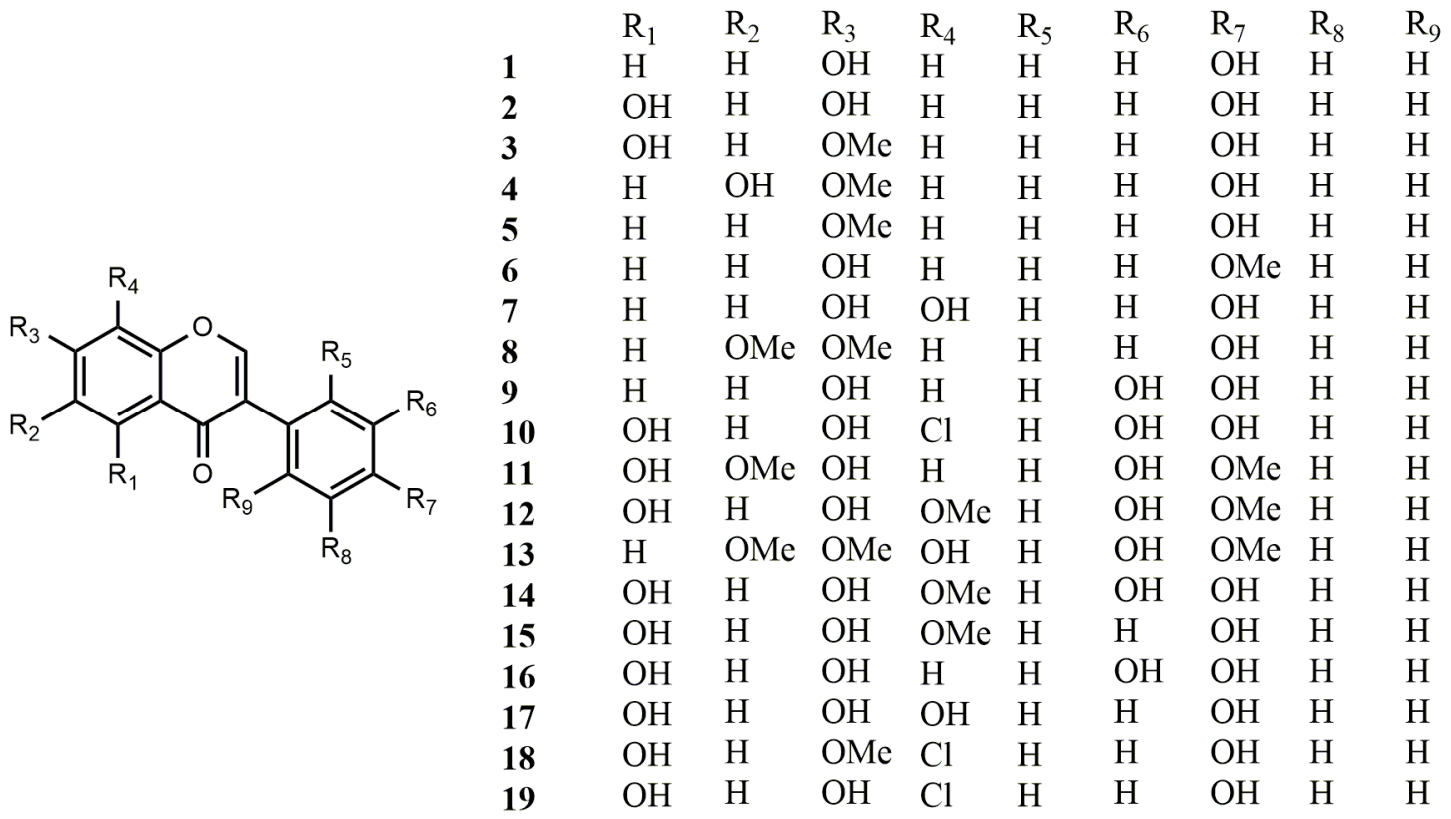

Figure 1. Reported structures of simple isoflavones 1-19 from fungi and actinomycetes. 
Molecules 2020, 25, 5112

3 of 27<smiles>[R]Cc1c([R])c([R5])c(-c2coc3c([R])c([R])c([R])c([R])c3c2=O)c([R6])c1[R6]</smiles>
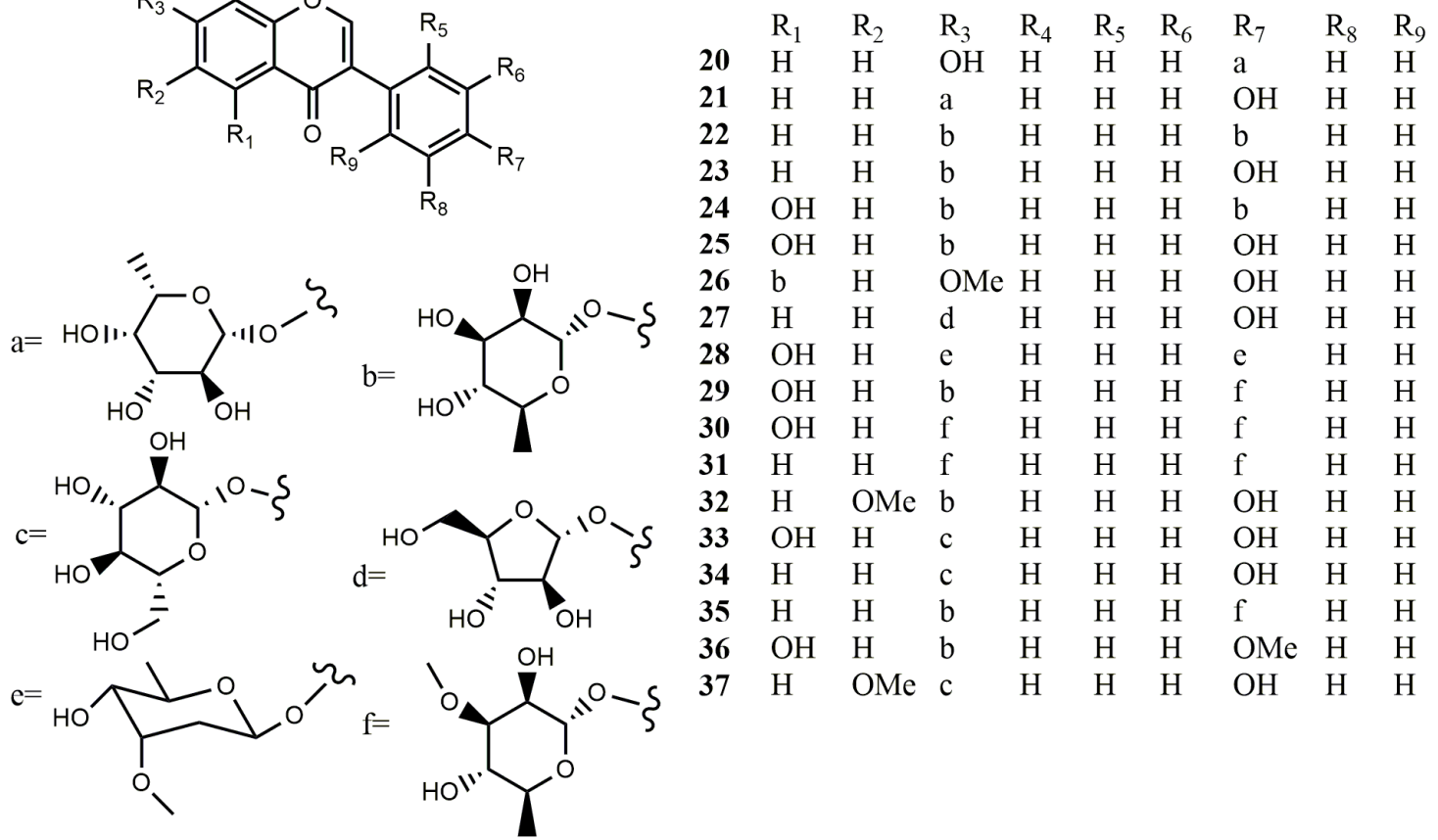

Figure 2. Reported structures of isoflavonoid glycosides 20-37 from fungi and actinomycetes.<smiles>CC(C)=CCc1c(O)cc2occ(-c3ccc(O)cc3O)c(=O)c2c1O</smiles><smiles>CC(C)(O)CCc1c(O)cc2occ(-c3ccc(O)cc3O)c(=O)c2c1O</smiles><smiles>CC(C)(O)C(O)Cc1c(O)cc2occ(-c3ccc(O)cc3O)c(=O)c2c1O</smiles>

38

39<smiles>CC(C)(O)C1Cc2c(cc3occ(-c4ccc(O)cc4O)c(=O)c3c2O)O1</smiles><smiles>CC1(C)Oc2cc3occ(-c4ccc(O)cc4O)c(=O)c3c(O)c2CC1O</smiles>

41

42<smiles>CC1(C)Oc2ccc(-c3coc4cc(O)cc(O)c4c3=O)c(O)c2CC1O</smiles><smiles>CC(C)(O)C1Cc2c(ccc(-c3coc4cc(O)cc(O)c4c3=O)c2O)O1</smiles><smiles></smiles><smiles>CC(C)=C(C)C1Cc2c(O)ccc(-c3coc4cc(O)cc(O)c4c3=O)c2O1</smiles><smiles>CC1(C)Oc2c(-c3coc4cc(O)cc(O)c4c3=O)ccc(O)c2CC1O</smiles><smiles>CC(C)(O)C(O)Cc1c(O)ccc(-c2coc3cc(O)cc(O)c3c2=O)c1O</smiles><smiles>Cc1cccc(O)c1C(=O)Oc1ccc(-c2coc3cc(O)cc(O)c3c2=O)cc1</smiles>

Figure 3. Reported structures of complex isoflavones 38-49 from fungi and actinomycetes. 


\subsubsection{Simple Isoflavonoids}

Four known isoflavonoids, daidzein (1), genistein (2), daidzein-7-O- $\alpha$-L-rhamnoside (23), and genistein-7-O- $\alpha$-L-rhamnoside (25) were derived from Indonesian actinomycete Streptomyces sp. TPU1401A. Compounds 1 and 2 could be transformed into 7-O-glycosides, daidzein-7-O- $\alpha$-L-rhamnoside and genistein-7-O- $\alpha$-L-rhamnoside by strain TPU1401A, respectively. The strain TPU1401A grew better when adding daidzein-7-O- $\alpha$-L-rhamnoside (23) and genistein-7-O- $\alpha$-L-rhamnoside (25) instead of compounds 1 and 2. This phenomenon suggested that the strain TPU1401A transformed isoflavones into isoflavone glycosides to hasten growth [32].

Five known isoflavones, genistein (2), prunetin (3), kakkatin (4), isoformononetin (5), and formononetin (6) were isolated from lichen-associated Amycolatopsis sp. YIM 130642. These compounds exhibited weak antimicrobial activities against several pathogens [33]. EtOH extracts of the fungal strain, Aspergillus sp. HK-388 afforded 8-hydroxydaidzein (7), which exhibited non-competitive inhibition on aldose reductase of human recombinant with its $K_{\mathrm{i}}$ value of $7.0 \mu \mathrm{M}$ [34].

During the chemical screening of a number of actinomycete strains from marine and terrestrial sources, six isoflavones were obtained from several Streptomyces strains and identified to be daidzein (1), genistein (2), 7-O-methylgenistein (3), 4',6-dihydroxy-7-methoxyisoflavone (4), 4'-hydroxy-6,7-dimethoxyisoflavone (8), 4',7-bis-( $\beta$-cymaropyranosyl)-genistein (28), and genistein-4'-(6"'-methyl)-salicylate (49), in which compounds 8, 28, and 49 are new compounds [35].

Three isoflavonoids, $4^{\prime}, 7,8$-trihydroxyisoflavone (7), $3^{\prime}, 4^{\prime}, 7$-trihydroxyisoflavone (9) and 8-chloro-3', 4', 5,7-tetrahydroxyisoflavone (10) were obtained from the culture broth of Streptomyces sp. OH-1049. These compounds showed antioxidant activity in vitro [36]. Compounds 9 and 10 were acetylated by adding pyridine and $\mathrm{Ac}_{2} \mathrm{O}$ to afford $3^{\prime}, 4^{\prime}, 7$-triacetoxyisoflavone and 8-chloro-3' $4^{\prime}, 5,7$-tetraacetoxyisoflavone, respectively [37].

Three new isoflavones, 3' ,5,7-trihydroxy-4',6-dimethoxyisoflavone (11), 3',5,7-trihydroxy-4' ${ }^{\prime}$, dimethoxyiso-flavone (12) and $3^{\prime}, 8$-dihydroxy-4' $4^{\prime}, 6,7$-trimethoxyisoflavone (13), were derived from Streptomyces culture filtrates. Compounds $\mathbf{1 1}$ and $\mathbf{1 2}$ were able to suppress the catechol-O-methyltransferase and dopa decarboxylase, and exhibit hypotensive action. Compound 13 was a specific inhibitor of catechol-O-methyltransferase [38]. A novel isoflavone, $3^{\prime}, 44^{\prime}, 5,7$-tetrahydroxy-8-methoxy (14), and four known compounds, genistein (2), psi-tectorigenin (15), orobol (16), and 8-hydroxygenistein (17) were obtained from the culture filtrates of fungi and streptomyces during the screening of inhibiting dopa decarboxylase. Compounds $\mathbf{1 4}$ and $\mathbf{1 6}$ exhibited remarkable activity in restraining dopa decarboxylase. Compound $\mathbf{1 6}$ also showed an excellent hypotensive effect on spontaneously hypertensive rats [39].

Four isoflavone glycosides and two isoflavones were isolated from Streptomyces xanthophaeus. They were determined as daidzein (1), genistein (2), daidzein-4',7-di- $\alpha$-L-rhamnoside (22), daidzein-7- $\alpha$-L-rhamnoside (23), genistein- $4^{\prime}, 7-\mathrm{di}-\alpha$-L-rhamnoside (24), and genistein 7- $\alpha$-L-rhamnoside (25). All of these compounds showed $\beta$-galactosidase inhibiting activities [40]. Ten isoflavone glycosides and one isoflavone, daidzein (1), were obtained from Streptomyces sp. RB1 associated with Macrotermes natalensis [17]. Later, seven isoflavone glycosides and one isoflavone, genistein (2) were additionally isolated from this strain [41]. Those glycosyl compounds are introduced in the following classification (Section 2.1.2). Eight known isoflavones, daidzein (1), genistein (2), 7-O-methyl genistein (3), kakkatin (4), 8-chlorogenistein (19), genistin (33), daidzin (34), glycitin (37), and a novel isoflavone named 7-O-methyl-8-chlorogenistein (18) were identified from Streptomyces strain YIM GS3536. The MIC values of $\mathbf{1 8}$ against C. albicans, E. coli, B. subtilis, and S. aureus were in the range of $23-35 \mu \mathrm{g} / \mathrm{mL}$. Compound 18 also showed appreciable cytotoxicity against human leukemia cell lines (HL60) and human melanoma cell lines (B16) with $\mathrm{IC}_{50}$ values of 19.9 and 17.5 $\mu \mathrm{g} / \mathrm{mL}$, respectively [42]. 


\subsubsection{Isoflavonoid Glycosides}

Three novel isoflavonoid glycosides, daidzein-4'-(2-deoxy- $\alpha$-L-fucopyranoside) (20), daidzein-7-(2-deoxy- $\alpha$-L-fucopyranoside) (21) and daidzein-4', 7 -di-(2-deoxy- $\alpha$-L-fucopyranoside) (71) were obtained from the culture broth of mangrove-derived actinomycete Micromonospora aurantiaca 110B. Though the new compounds were unable to inhibit pathogenic fungus or bacteria, they exhibited moderate cytotoxic activities to the human colon tumor cell line HCT116, the human lung carcinoma cell line A549, and hepatocellular liver carcinoma cell line HepG2 [43]. Four isoflavone glycosides were obtained from S. xanthophaeus. They were identified as daidzein-4',7-di- $\alpha$-L-rhamnoside (22), daidzein-7- $\alpha$-L-rhamnoside (23), genistein- $4^{\prime}, 7-d i-\alpha$-Lrhamnoside (24), and genistein-7- $\alpha$-L-rhamnoside (25). All of these compounds had $\beta$-galactosidase inhibiting activities [40].

From the fermentation broth of the lichen-associated Amycolatopsis sp. YIM 130642, a new isoflavonoid glycoside, 7-O-methyl-5-O- $\alpha$-L-rhamnopyranosylgenistein (26), along with a firstly natural occurring isoflavonoid glycoside, 7-O- $\alpha$-D-arabinofuranosyl daidzein (27) was isolated. Compound 26 showed moderate inhibitory activity towards E. coli and S. aureus with MIC value of $64 \mu \mathrm{g} / \mathrm{mL}$ [33]. A new isoflavone glycoside, 4' 7 -bis-( $\beta$-cymaropyranosyl)-genistein (28) was isolated from Streptomyces sp. HKI 129-L [35].

Ten isoflavonoid glycosides were isolated from Streptomyces sp. RB1 originating from $M$. natalensis, including three novel ones, termisoflavones A-C (29-31) and seven known compounds, daidzein- $4^{\prime}, 7$-di- $\alpha$-L-rhamnoside (22), daidzein-7- $\alpha$-L-rhamnoside (23), genistein- $4^{\prime}, 7$-di- $\alpha$-Lrhamnoside (24), genistein-7- $\alpha$-L-rhamnoside (25), 6-O-methyl-7-O- $\alpha$-L-rhamnopyranosyldaidzein (32), genistin (33), and daidzin (34). Unfortunately, these compounds showed no antimicrobial activities. Only compounds $\mathbf{2 3}$ and $\mathbf{3 4}$ showed protective effects on kidney cells [17].

One new isoflavonoid glycoside, termisoflavone D (35), together with six known analogues, daidzein-4', 7-di- $\alpha$-L-rhamnopyranoside (22), daidzein-7- $\alpha$-L-rhamnopyranoside (23), genistein-4 $4^{\prime}, 7-\mathrm{di}-\alpha$-L-rhamnopyranoside (24), genistein-7- $\alpha$-D-glucopyranoside (33), daidzein-7- $\alpha$-Dglucopyranoside (34), and 4'-O-methyl-7-O- $\alpha$-L-rhamnopyranosylgenistein (36), were identified from Streptomyces sp. RB1. Compound 23 prevented glutamate-induced HT22 cell death by blocking the accumulation of intracellular reactive oxygen species (ROS) [41]. Three isoflavone glycosides, genistin (33), daidzin (34), and glycitin (37), were derived from Streptomyces strain YIM GS3536 [42]. Daidzein-7-O- $\alpha$-L-rhamnoside (23) and genistein-7-O- $\alpha$-L-rhamnoside (25) were derived from Indonesian actinomycete Streptomyces sp. TPU1401A [32].

\subsubsection{Complex Isoflavones}

An antifungal luteone, 5,7,2', 4' -tetrahydroxy-6-(3,3-dimethylallyl) isoflavone (38), was obtained from the cultures of Aspergillus flavus and Botrytis cinerea. At the level of $7-14 \mu \mathrm{g} / \mathrm{cm}^{2}$, the growth of Chlamydia herbarum was completely inhibited by compound 38 [44]. Four compounds, luteone hydrate (39), luteone metabolite AF-2 (40), BC-1 (41), and BC-2 (42), were obtained from the co-cultures of A. flavus and B. cinerea. Five isoflavonoids, M-1-1 (44), M-1-2 (45), M-2 (46), M-3-1 (47), and M-3-2 (48) were isolated from the fungus $A$. flavus or B. cinereal by converting licoisoflavone A (43). When their inhibitory ability to grow Cladosporium herbarum was tested, compounds $\mathbf{3 8}$ and $\mathbf{4 3}$ were found to completely inhibit growth at the level of $25-100 \mu \mathrm{g} / \mathrm{cm}^{2}$, while compound 44 had only a small inhibition zone at the level of $100 \mu \mathrm{g} / \mathrm{cm}^{2}$ [45]. A new isoflavone, genistein- $4^{\prime}$-(6"-methyl)-salicylate (49) was obtained from Streptomyces sp. GW27/2506 [35].

\subsection{Flavonoids}

Flavonoid derivatives are natural products commonly found in medicinal plants and are synthesised from phenylpropanoid and acetate-derived precursors [11]. Some flavonoids have also been discovered from fungi and actinomycetes. We also classified these flavonoids into three 
groups: simple flavonoids, flavonoid glycosides, and complex flavonoids, and the structures of the compounds are shown in Figures 4-6, respectively. Most of these compounds mainly exhibit antioxidant, antimicrobial, anti-inflammatory, antitumour, antifouling, and $\alpha$-glucosidase inhibitory activity.

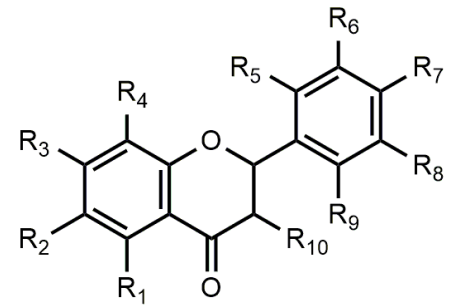

$\begin{array}{lllllllllll} & \mathrm{R}_{1} & \mathrm{R}_{2} & \mathrm{R}_{3} & \mathrm{R}_{4} & \mathrm{R}_{5} & \mathrm{R}_{6} & \mathrm{R}_{7} & \mathrm{R}_{8} & \mathrm{R}_{9} & \mathrm{R}_{10} \\ \mathbf{5 0} & \mathrm{OH} & \mathrm{H} & \mathrm{OH} & \mathrm{H} & \mathrm{H} & \mathrm{OH} & \mathrm{OH} & \mathrm{OH} & \mathrm{H} & \mathrm{OH} \\ \mathbf{5 2} & \mathrm{OMe} & \mathrm{H} & \mathrm{OMe} & \mathrm{H} & \mathrm{H} & \mathrm{H} & \mathrm{OH} & \mathrm{H} & \mathrm{H} & \mathrm{H} \\ \mathbf{5 3} & \mathrm{OMe} & \mathrm{H} & \mathrm{OH} & \mathrm{H} & \mathrm{H} & \mathrm{H} & \mathrm{OH} & \mathrm{H} & \mathrm{H} & \mathrm{H} \\ \mathbf{5 4} & \mathrm{OH} & \mathrm{H} & \mathrm{OH} & \mathrm{H} & \mathrm{H} & \mathrm{H} & \mathrm{H} & \mathrm{H} & \mathrm{H} & \mathrm{H} \\ \mathbf{6 4} & \mathrm{OH} & \mathrm{H} & \mathrm{OH} & \mathrm{H} & \mathrm{H} & \mathrm{H} & \mathrm{OH} & \mathrm{H} & \mathrm{H} & \mathrm{Me}\end{array}$<smiles>[R]c1c([R])c([R])c(-c2oc3c([R])c([R])c([R])c([R])c3c(=O)c2[R6])c([R])c1[R]</smiles>

$\begin{array}{llllllllll}\mathrm{R}_{1} & \mathrm{R}_{2} & \mathrm{R}_{3} & \mathrm{R}_{4} & \mathrm{R}_{5} & \mathrm{R}_{6} & \mathrm{R}_{7} & \mathrm{R}_{8} & \mathrm{R}_{9} & \mathrm{R}_{10}\end{array}$ $\begin{array}{lllllllllll}\mathbf{5 1} & \mathrm{OH} & \mathrm{H} & \mathrm{OH} & \mathrm{H} & \mathrm{H} & \mathrm{H} & \mathrm{OH} & \mathrm{OMe} & \mathrm{H} & \mathrm{OH}\end{array}$ $\begin{array}{lllllllllll}56 & \mathrm{OH} & \mathrm{H} & \mathrm{OMe} & \mathrm{OMe} & \mathrm{H} & \mathrm{H} & \mathrm{H} & \mathrm{H} & \mathrm{OH} & \mathrm{OMe}\end{array}$ $\begin{array}{lllllllllll}57 & \mathrm{H} & \mathrm{OH} & \mathrm{H} & \mathrm{OH} & \mathrm{H} & \mathrm{OMe} & \mathrm{H} & \mathrm{OH} & \mathrm{OH} & \mathrm{H}\end{array}$ $\begin{array}{llllllllllll}58 & \mathrm{OH} & \mathrm{H} & \mathrm{OMe} & \mathrm{OMe} & \mathrm{OH} & \mathrm{Cl} & \mathrm{H} & \mathrm{H} & \mathrm{H} & \mathrm{OMe}\end{array}$ 59 OMe H

$60 \mathrm{OMe} H$

$61 \quad \mathrm{OH} \quad \mathrm{H}$ $\mathrm{OMe} \mathrm{OMe} \mathrm{H} \quad \mathrm{H} \quad \mathrm{H}$ $\begin{array}{llllllll}\mathrm{OMe} & \mathrm{OMe} & \mathrm{H} & \mathrm{H} & \mathrm{H} & \mathrm{H} & \mathrm{OH} & \mathrm{OMe}\end{array}$<smiles>Oc1cc(O)c2c(c1)CC(O)C(c1ccc(O)c(O)c1)O2</smiles>

55

Figure 4. Reported structures of simple flavonoids 50-66 from fungi and actinomycetes.

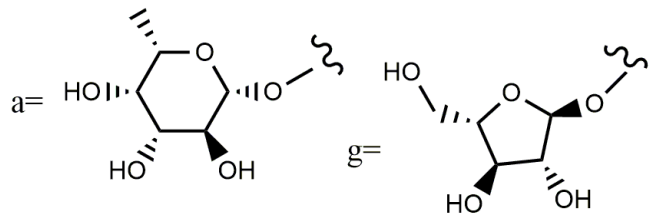<smiles>[Y6]O[C@H]1O[C@H](CO)[C@@H](O)[C@H](O)[C@H]1O[Te]</smiles>

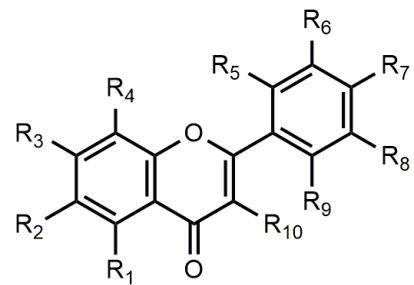

$\begin{array}{lllllllllll}\mathbf{6 7} & \mathrm{R}_{1} & \mathrm{R}_{2} & \mathrm{R}_{3} & \mathrm{R}_{4} & \mathrm{R}_{5} & \mathrm{R}_{6} & \mathrm{R}_{7} & \mathrm{R}_{8} & \mathrm{R}_{9} & \mathrm{R}_{10} \\ \mathbf{6 8} & \mathrm{OH} & \mathrm{H} & \mathrm{OH} & \mathrm{H} & \mathrm{H} & \mathrm{OH} & \mathrm{OH} & \mathrm{H} & \mathrm{H} & \mathrm{g} \\ \mathbf{6 9} & \mathrm{OH} & \mathrm{H} & \mathrm{OH} & \mathrm{H} & \mathrm{H} & \mathrm{OH} & \mathrm{OH} & \mathrm{H} & \mathrm{H} & \mathrm{H} \\ \mathbf{7 1} & \mathrm{H} & \mathrm{H} & \mathrm{a} & \mathrm{H} & \mathrm{H} & \mathrm{OH} & \mathrm{OH} & \mathrm{H} & \mathrm{H} & \mathrm{i} \\ \mathbf{7 3} & \mathrm{OH} & \mathrm{H} & \mathrm{OH} & \mathrm{H} & \mathrm{H} & \mathrm{OH} & \mathrm{OH} & \mathrm{H} & \mathrm{H} & \mathrm{H} \\ \mathbf{7 4} & \mathrm{OH} & \mathrm{H} & \mathrm{OH} & \mathrm{H} & \mathrm{H} & \mathrm{OH} & \mathrm{OH} & \mathrm{H} & \mathrm{H} & \mathrm{j}\end{array}$<smiles>CC(O)C(C)C(=O)NC1CC(Oc2cc(CO)c3c(=O)cc(-c4ccccc4)oc3c2O)C(C)OC1O</smiles>

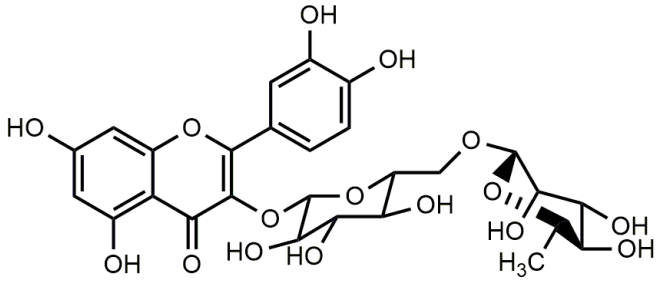

72

Figure 5. Reported structures of flavonoid glycosides 67-74 from fungi and actinomycetes. 


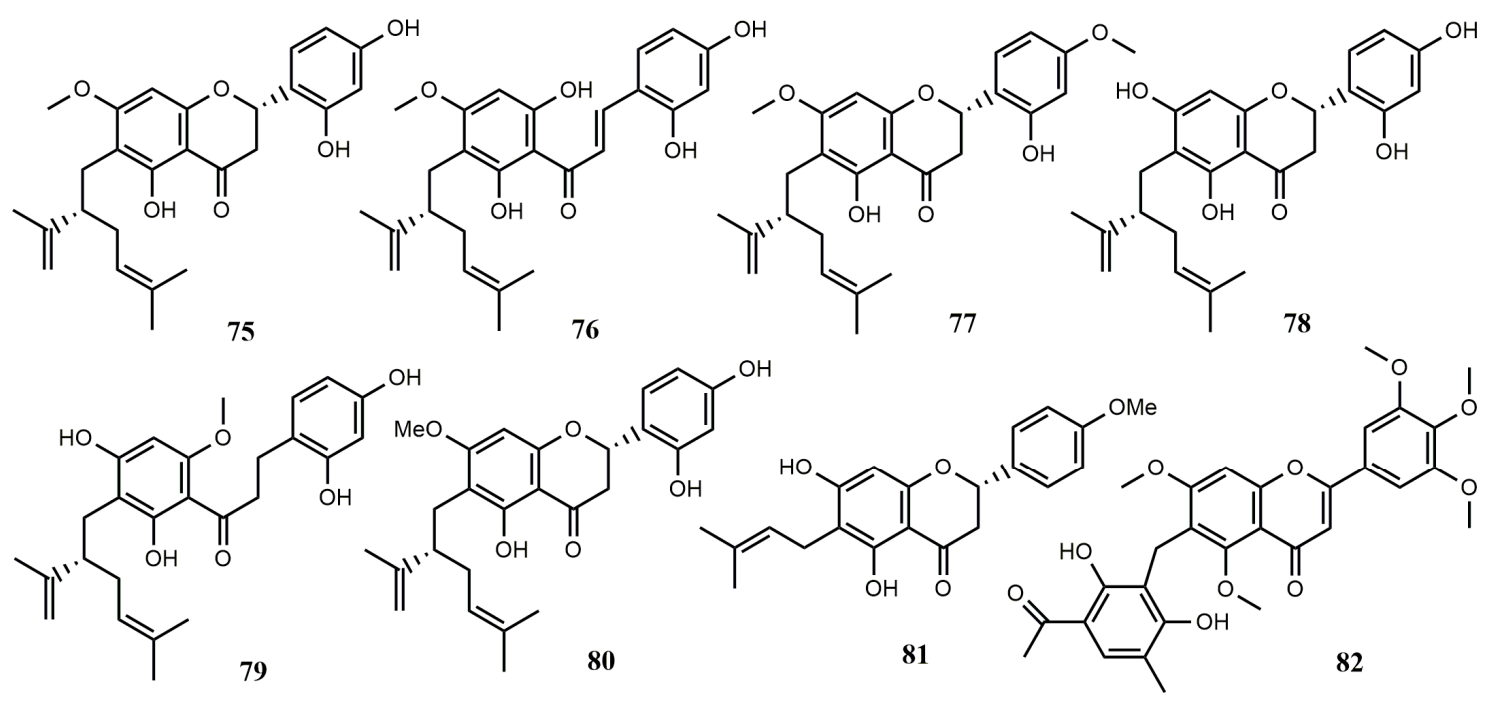

Figure 6. Reported structures of complex flavonoids 67-74 from fungi and actinomycetes.

\subsubsection{Simple Flavonoids}

Trichoderma strains are widely used to inhibit pathogens and promote the growth of plants. LC-QQQ-MS showed that three simple flavonoids, dihydromyricetin (50), isorhamnetin (51), and 4-hydroxy-5,7-dimethoxyflavanone (52), existed in the culture broth of fungus T. asperellum TJ01, and their proportion was the highest when cultured at $72 \mathrm{~h}$. [46].

Three flavonoids, named 7-O-methylnaringenin (53), pinocembrin (54), and (-)-epicatechin (55), were isolated from the endophytic fungus Annulohypoxylon elevatidiscus BCRC 34014, which was isolated from decorticated woods. It is worth mentioning that this is the first time that flavonoids have been found in this species [47].

The endophytic fungus Xylaria papulis BCRC 09F0222 was isolated from hairy woody plants. Three flavonoids, named myricetin (50), myricitrin (73), and quercitrin (74), were obtained from this strain [48]. Since the fungus was cultivated on rice, which is a feeding plant known to produce myricetin and other flavonoids [49], these metabolites might be derived from rice or synthesized by modifying the existing flavonoid precursors in culture medium. Dechlorochlorflavonin (56), a known flavonoid derivative, was isolated from the fungus Aspergillus candidus Bdf-2 derived from insects. Compound $\mathbf{5 6}$ was assessed for antibacterial activities against Ralstonia solanacearum and Staphylococcus aureus ATCC29213 with MIC values of $64 \mu \mathrm{g} / \mathrm{mL}$. However, no antioxidant activity was detected [50].

A new flavonoid, named 6,8,5', $6^{\prime}$-tetrahydroxy-3'-methylflavone (57), was obtained from the fungal strain Penicillium sp. SCSGAF 0023. This strain could help the host gorgonian corals against marine pathogenic bacteria, biological pollution organisms, and other invaders by producing antifouling and antibacterial compounds. Compound $\mathbf{5 7}$ had potent antifouling activity against Balanus amphitrite larvae settlement with an $\mathrm{EC}_{50}$ value of $6.7 \mu \mathrm{g} / \mathrm{mL}$ [51]. A new antifungal antibiotic, 3'-chloro-2',5-dihydroxy-3,7,8-trimethoxy flavone (58) was isolated from A. candidus strains ATCC 20022 and CMI 16046 [52]. Two new flavonoids named aspergivones A (59) and B (60) were isolated from the fungus $A$. candidus. The strain was isolated from the gorgonian coral Anthogorgia ochracea, which was collected from the South China Sea. Compound $\mathbf{6 0}$ displayed only weak inhibitory activity against $\alpha$-glucosidase with an $\mathrm{IC}_{50}$ value of $244 \mu \mathrm{g} / \mathrm{mL}$. Cytotoxicity and antibacterial activity were not showed for compound 59 [53].

In addition, chlorflavonin (58), 3'-bromo-2',5-dihydroxy-3,7,8-trimethoxyflavone (61), and dechlorochlorflavonin (62) were isolated from the fermentation broth of the fungus Acanthostigmella sp. CL12082. Compound $\mathbf{6 1}$ showed strong antifungal activity against Aspergillus fumigatus and Candida albicans with $\mathrm{IC}_{50}$ values of 0.54 and $0.11 \mu \mathrm{g} / \mathrm{mL}$. Compound 58 displayed significant activity against the growth of pathogenic fungi, C. albicans and A. fusigatus with $\mathrm{IC}_{50}$ values of 0.035 and $0.10 \mu \mathrm{g} / \mathrm{mL}$, 
as well as against the growth of HeLa cells $\mathrm{IC}_{50}$ values of $20 \mu \mathrm{g} / \mathrm{mL}$. At the same time, these three compounds showed weak inhibition of growth of pathogenic fungi, Cryptococus neoformans with $\mathrm{IC}_{50}$ values of 20,12, and $16 \mu \mathrm{g} / \mathrm{mL}$, respectively [54]. Quercetin (63) was produced by an endophytic fungi Psathyrella candolleana from the seed of Ginkgo biloba. It displayed antibacterial activity against $S$. aureus with MIC values of $0.3906 \mathrm{mg} / \mathrm{mL}$ [55].

A study reported the isolation and determination of WS7528 (64), produced by Streptomyces sp. No. 7528 derived from a soil sample obtained at Nara Prefecture, Japan. It was tested orally and subcutaneously in immature rats to verify its effect on the growth of the uterus, which had also weak anti-inflammatory activity and could induce growth of the cell line MCF-7 [56]. Two flavonoids, rhamnazin (65) and cirsimaritin (66), obtained from microbial sources for the first time, were obtained from protoplast fusion between Streptomyces strains Merv 1996 and Merv 7409. Compound 65 showed remarkable activities against filamentous fungi $B$. fabae and $A$. niger with concentrations of 2.5 and 1.0 $\mu \mathrm{g} / \mathrm{mL}$, respectively. Compound $\mathbf{6 6}$ showed a strong antifungal activity in vitro against $C$. neoformans, $C$. albicans, Pichia angusta, and Rhodotorula minuta with MIC value of $1 \mu \mathrm{g} / \mathrm{mL}$, while showed no inhibitory effect against filamentous fungi [57].

\subsubsection{Flavonoid Glycosides}

Flavonoid glycosides derived from endophytic fungi were reported for the first time in 2016. Three quercetin-3-O-glycosides, named guaijaverin (67), isoquercitrin (68), and hyperin (69), were isolated from the endophytic fungus Nigrospora oryzae. The fungus was isolated from the leaves of the Nigerian mistletoe Loranthus micranthus, which was widely used in African traditional medicine [16]. Actinoflavoside (70), possessing a rare 2,3,6-trideoxy-3-amino-ribopyranoside aminosugar skeleton, was obtained from the fermentation broth of a marine Streptomyces sp. [58]. A flavonoid glycoside, daidzein-4' ${ }^{\prime}$ 7-di-(2-deoxy- $\alpha$-L-fucopyranoside) (71), was obtained from actinomycetes, which showed moderate activity against tumor cells of A549, HepG2, and HCT116 [43].

The strain Streptomyces sp. ERINLG-4 was isolated from the soil samples collected from a depth of $5-15 \mathrm{~cm}$ in the Doddabetta forest. The EtOAc extract of the strain showed potent cytotoxic activity in vitro against the A549 lung adenocarcinoma cancer cell line. The following work led to the isolation of an active component, quercetin-3-O- $\beta$-L-rhamnopyranosyl- $(1 \rightarrow 6)-\beta$-D-glucopyranoside (72), showing prominent cytotoxic activity against the A549 lung cancer cell line with an $\mathrm{IC}_{50}$ value of $82 \mu \mathrm{g} / \mathrm{mL}$. However, it showed no toxicity against the Vero normal cell line, up to $2000 \mu \mathrm{g} / \mathrm{mL}$ [59]. Three flavonoids, named myricetin (50), myricitrin (73), and quercitrin (74) were obtained from the endophytic fungus X. papulis BCRC 09F0222 isolated from hairy woody plants [48].

\subsubsection{Complex Flavonoids}

Two new lavandulylated flavonoids, 6-lavandulyl-7-methoxy-5,2', $4^{\prime}$-trihydroxylflavanone (75) and 5'-lavandulyl-4'-methoxy-2,4,2',6'-tetrahydroxylchalcone (76) were isolated from the sponge-derived actinomycete Streptomyces sp. G246. Compounds 75 and $\mathbf{7 6}$ showed a broad spectrum of antimicrobial activity. Compound $\mathbf{7 6}$ showed excellent inhibitory effects on C. albicans, S. aureus, Bacillus cereus, Enterococcus faecalis, Salmonella enterica, and Pseudomonas aeruginosa, but no inhibitory effect on Escherichia coli. [60].

Three new lavandulylated flavonoids, (2S,2"S)-6-lavandulyl-7,4'-dimethoxy-5,2' dihydroxylflavanone (77), (2S,2"S)-6-lavandulyl-5,7,2', $4^{\prime}$-tetrahydroxylflavanone (78) and (2"S)-5'-lavandulyl-2'-methoxy-2,4,4',6'-tetrahydroxylchalcone (79), together with two known compounds, (2S,2"S)-6-lavandulyl-7-methoxy-5,2', $4^{\prime}$-trihydroxyl-flavanone (80) and 6-prenyl-4'-methoxy-5,7-dihydroxylflavanone (81), were isolated from the fermentation broth of Streptomyces sp. G248. Compounds 77-79 showed remarkable antimicrobial activity, and compounds 80 and $\mathbf{8 1}$ were found to inhibit Mycobacterium tuberculosis H37Rv with the MIC values of 6.0 and $11.1 \mu \mathrm{g} / \mathrm{mL}$ [61]. A strain of Penicillium griseoroseum was isolated from Coffeea arabica seeds, and its 
metabolites were studied in $5,7,3^{\prime}, 4^{\prime}, 5^{\prime}$-pentamethoxyflavanone supplemented medium to obtain bezylated flavanone (82) [62].

\section{Microbiological Transformation of Isoflavonoids and Flavonoids}

Biotransformation is a well-known process of effectively obtaining isoflavones and flavonoids. The main reactions in the conversion process include hydroxylation, methylation, glycosylation, and cyclisation, through which some of the rare or expensive isoflavones and flavonoids can be obtained $[63,64]$. In this section, we review the biotransformation of isoflavones and flavonoids by fungi and actinomycetes, and the compounds involved in the microbiological transformation are shown in Figures 7 and 8, respectively.

\subsection{Biotransformation of Isoflavones and Flavonoids by Fungi}

In order to obtain O-dihydroxyisoflavones, Aspergillus saito $i$ was used for transforming daidzein (1) and genistin (33) to generate 8-hydroxydaidzein (7) and 8-hydroxygenistein (17), respectively, by deglycosylation and hydroxylation [65].

Three isoflavones were used to study the biotransformation by Aspergillus niger, and it was found that daidzein (1) was not metabolized. The C-6 position of 7,4'-dimethoxyisoflavone (83) was hydroxylated to form 6-hydroxy-7, $4^{\prime}$-dimethoxyisoflavone (85), which was then converted into 1 by demethylation of C-7 and C-4' positions. In addition, 7,4'-diacetoxyisoflavone (84) was converted into 1 through the hydrolysis of C-7 and C-4' positions [66]. 6,7,4'-Trimethoxyisoflavone (86) and 5,7,4'-trimethoxyisoflavone (87) were studied for their biotransformation by $A$. niger. They could be converted to $4^{\prime}$-hydroxy-6,7-dimethoxyisoflavone (8) and 4'-hydroxy-5,7-dimethoxyisoflavone (88), respectively, by demethylation at C-4' position [67].

The isoflavone, luteone [5,7,2', $4^{\prime}$-tetrahydroxy-6-(3,3-dimethylallyl) isoflavone] (38) could be transformed into 2",3"-dihydro-3"-hydroxyluteone (89), 2",3"-dihydrodihydroxyluteone (90), dihydrofuranoisoflavone (41) and dihydropyranoisoflavone (91) by cultures of A. flavus and B. cinerea. The main products $\mathbf{4 1}$ and $\mathbf{8 9}$ were less toxic to $C$. herbarum than compound $\mathbf{3 8}$ [44]. Two isoflavones, 7-O-methyl-2,3-dehydrokievitone epoxide (93) and 7-O-methyl-2,3-dehydrokievitone glycol (94), were formed from 2,3-dehydrokievitone (92) by methylating with ethereal diazomethane under the metabolism by $B$. cinerea. The epoxide was regarded as the key metabolic intermediate involved in the transformation from precursor compounds containing a prenyl side-chain with ortho-hydroxylation to 2,3-dihydroxy-3-methylbutyl-substituted isoflavones [68].

Biotransformation of two flavonoids was studied by using four strains of $A$. niger, three of which were mutated by ultraviolet irradiation. Flavanone (95) and 6-hydroxyflavanone (96) were transformed to flavan-4-ol (99) and 6-hydroxyflavan-4-ol (101), respectively, through decarbonylation, and the dehydrogenation at C-2 and C-3 positions produced flavone (97) and 6-hydroxyflavone (102), respectively. Compound 95 was reduced at C-4 and hydroxylated at C-7 to form 7-hydroxyflavan-4-ol (100), and dehydrogenation at C-2 and C-3, along with hydroxylation at C-3 to from 3-hydroxyflavone (98) [69].

2", 3"-Dihydro-3"-hydroxywighteone (106) was obtained by biotransformation from the antifungal compound, wighteone [5,7,4' -trihydroxy-6-(3,3-dimethylallyl) isoflavone] (103) under the modification of $A$. flavus and B. cinerea and the latter was further transformed into dihydrofurano-isoflavone (104). Small amounts of dihydropyrano-isoflavone (105), dihydrofurano-isoflavone (104), and 2",3"-dihydrodihydroxywighteone (107) were obtained in A. flavus culture medium. Compounds 105 and 107 were obtained in B. cinerea medium [70]. 
<smiles>[R]c1c([R])c([R])c(-c2coc3c([R])c([R])c([R])c([R])c3c2=O)c([R])c1[R]</smiles><smiles>[R]c1c([R])c([R])c(C2CC(=O)c3c([R])c([R])c([R])c([R])c3O2)c([R])c1[R]</smiles><smiles>OC[C@H]1O[C@@H](OS)[C@@H](O)[C@H](O)[C@@H]1O</smiles><smiles>OC[C@H]1O[C@H](O[Te])[C@@H](O)[C@H](O)[C@H]1O</smiles><smiles>CO[C@H]1O[C@H](CO)[C@@H](O[Te])[C@H](O)[C@H]1O</smiles>

$\begin{array}{llllllllll}83 & \mathrm{H} & \mathrm{H} & \mathrm{OMe} & \mathrm{H} & \mathrm{H} & \mathrm{H} & \mathrm{OMe} & \mathrm{H} & \mathrm{R}\end{array}$

$\begin{array}{llllllllll}84 & \mathrm{H} & \mathrm{H} & \mathrm{OAc} & \mathrm{H} & \mathrm{H} & \mathrm{H} & \mathrm{OAc} & \mathrm{H} & \mathrm{H}\end{array}$

$\begin{array}{llllllllll}85 & \mathrm{H} & \mathrm{OH} & \mathrm{OMe} & \mathrm{H} & \mathrm{H} & \mathrm{H} & \mathrm{OMe} & \mathrm{H} & \mathrm{H}\end{array}$

$\begin{array}{llllllllll}86 & \mathrm{H} & \mathrm{OMe} & \mathrm{OMe} & \mathrm{H} & \mathrm{H} & \mathrm{H} & \mathrm{OMe} & \mathrm{H} & \mathrm{H}\end{array}$

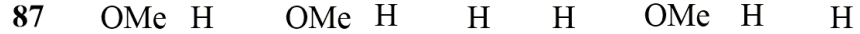

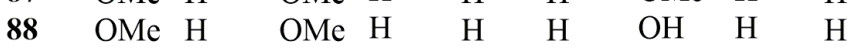

$\begin{array}{llllllllll}108 & \mathrm{H} & \mathrm{H} & \mathrm{OH} & \mathrm{c} & \mathrm{H} & \mathrm{H} & \mathrm{OH} & \mathrm{H} & \mathrm{H}\end{array}$

$\begin{array}{llllllllll}109 & \mathrm{H} & \mathrm{H} & \mathrm{OH} & \mathrm{c} & \mathrm{H} & \mathrm{OH} & \mathrm{OH} & \mathrm{H} & \mathrm{H}\end{array}$

$\begin{array}{llllllllll}162 & \mathrm{H} & \mathrm{H} & \mathrm{k} & \mathrm{H} & \mathrm{H} & \mathrm{H} & \mathrm{OH} & \mathrm{H} & \mathrm{H}\end{array}$

$199 \begin{array}{lllllllll}\mathrm{H} & \mathrm{H} & \mathrm{k} & \mathrm{H} & \mathrm{H} & \mathrm{H} & \mathrm{OMe} & \mathrm{H} & \mathrm{H}\end{array}$

$\begin{array}{lllllllll}\mathrm{R}_{1} & \mathrm{R}_{2} & \mathrm{R}_{3} & \mathrm{R}_{4} & \mathrm{R}_{5} & \mathrm{R}_{6} & \mathrm{R}_{7} & \mathrm{R}_{8} & \mathrm{R}_{9}\end{array}$

$\begin{array}{llllllllll}95 & \mathrm{H} & \mathrm{H} & \mathrm{H} & \mathrm{H} & \mathrm{H} & \mathrm{H} & \mathrm{H} & \mathrm{H} & \mathrm{H}\end{array}$

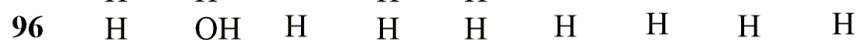

$114 \begin{array}{lllllllll}\mathrm{H} & \mathrm{H} & \mathrm{H} & \mathrm{H} & \mathrm{H} & \mathrm{H} & \mathrm{OH} & \mathrm{H} & \mathrm{H}\end{array}$

$\begin{array}{llllllllll}116 & \mathrm{OH} & \mathrm{H} & \mathrm{OH} & \mathrm{H} & \mathrm{H} & \mathrm{H} & \mathrm{OH} & \mathrm{H} & \mathrm{H}\end{array}$

$\begin{array}{llllllllll}123 & \mathrm{H} & \mathrm{H} & \mathrm{OH} & \mathrm{H} & \mathrm{H} & \mathrm{H} & \mathrm{H} & \mathrm{H} & \mathrm{H}\end{array}$

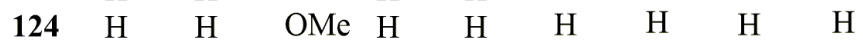

$\begin{array}{llllllllll}125 & \mathrm{H} & \mathrm{H} & \mathrm{OMe} & \mathrm{H} & \mathrm{H} & \mathrm{OH} & \mathrm{OH} & \mathrm{H} & \mathrm{H}\end{array}$

$\begin{array}{llllllllll}166 & \mathrm{H} & \mathrm{H} & \mathrm{H} & \mathrm{H} & \mathrm{OMe} & \mathrm{H} & \mathrm{H} & \mathrm{H} & \mathrm{H}\end{array}$

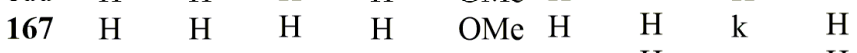

$\begin{array}{llllllllll}168 & \mathrm{H} & \mathrm{H} & \mathrm{H} & \mathrm{H} & \mathrm{k} & \mathrm{H} & \mathrm{H} & \mathrm{H} & \mathrm{H}\end{array}$

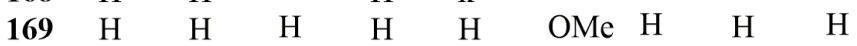

$\begin{array}{llllllllll}171 & \mathrm{H} & \mathrm{k} & \mathrm{H} & \mathrm{H} & \mathrm{H} & \mathrm{OH} & \mathrm{H} & \mathrm{H} & \mathrm{H}\end{array}$

$\begin{array}{llllllllll}172 & \mathrm{H} & \mathrm{H} & \mathrm{H} & \mathrm{H} & \mathrm{H} & \mathrm{H} & \mathrm{OMe} & \mathrm{H} & \mathrm{H}\end{array}$

$\begin{array}{llllllllll}173 & \mathrm{H} & \mathrm{H} & \mathrm{H} & \mathrm{H} & \mathrm{H} & \mathrm{H} & \mathrm{k} & \mathrm{H} & \mathrm{H}\end{array}$

$\begin{array}{llllllllll}174 & \mathrm{H} & \mathrm{k} & \mathrm{H} & \mathrm{H} & \mathrm{H} & \mathrm{H} & \mathrm{OH} & \mathrm{H} & \mathrm{H}\end{array}$

$\begin{array}{llllllllll}175 & \mathrm{H} & \mathrm{k} & \mathrm{H} & \mathrm{H} & \mathrm{H} & \mathrm{OH} & \mathrm{OH} & \mathrm{H} & \mathrm{H}\end{array}$

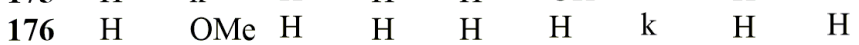

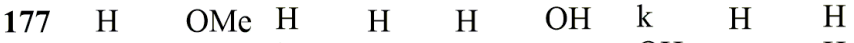

$\begin{array}{llllllllll}190 & \mathrm{OH} & \mathrm{H} & \mathrm{k} & \mathrm{H} & \mathrm{H} & \mathrm{H} & \mathrm{OH} & \mathrm{H} & \mathrm{H}\end{array}$

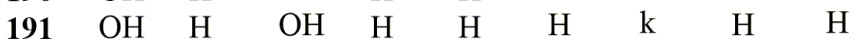

$\begin{array}{llllllllll}192 & \mathrm{OH} & \mathrm{H} & \mathrm{OH} & \mathrm{H} & \mathrm{H} & \mathrm{H} & \mathrm{h} & \mathrm{H} & \mathrm{H}\end{array}$

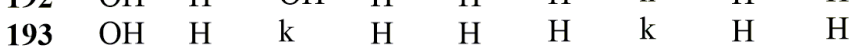

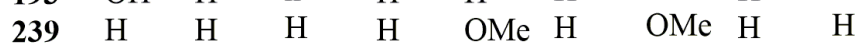

$\begin{array}{llllllllll}240 & \mathrm{H} & \mathrm{H} & \mathrm{H} & \mathrm{H} & \mathrm{OMe} & \mathrm{H} & \mathrm{OH} & \mathrm{H} & \mathrm{H}\end{array}$

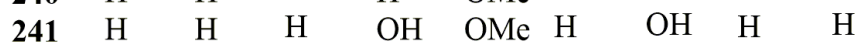

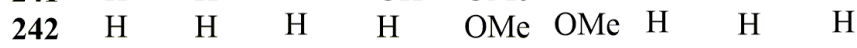

$\begin{array}{llllllllll}243 & \mathrm{H} & \mathrm{H} & \mathrm{H} & \mathrm{H} & \mathrm{OMe} & \mathrm{OH} & \mathrm{H} & \mathrm{H} & \mathrm{H}\end{array}$

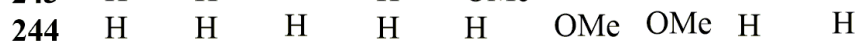

$\begin{array}{llllllllll}245 & \mathrm{H} & \mathrm{H} & \mathrm{H} & \mathrm{H} & \mathrm{H} & \mathrm{OH} & \mathrm{OH} & \mathrm{H} & \mathrm{H}\end{array}$

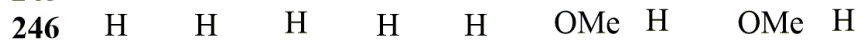

$\begin{array}{llllllllll}247 & \mathrm{H} & \mathrm{H} & \mathrm{H} & \mathrm{H} & \mathrm{H} & \mathrm{OH} & \mathrm{H} & \mathrm{OMe} & \mathrm{H}\end{array}$

$\begin{array}{llllllllllll}248 & \mathrm{H} & \mathrm{H} & \mathrm{OMe} & \mathrm{H} & \mathrm{OMe} & \mathrm{OMe} & \mathrm{H} & \mathrm{H} & \mathrm{H}\end{array}$

$\begin{array}{lllllllllll}249 & \mathrm{H} & \mathrm{H} & \mathrm{OMe} & \mathrm{H} & \mathrm{OMe} & \mathrm{OH} & \mathrm{H} & \mathrm{H} & \mathrm{H}\end{array}$

250 H $\mathrm{H} \quad \mathrm{OMe} H \quad$ OMe $\mathrm{H}$ OMe $\mathrm{H} \quad \mathrm{H}$

$\begin{array}{llllllllll}251 & \mathrm{H} & \mathrm{H} & \mathrm{OMe} & \mathrm{H} & \mathrm{OH} & \mathrm{H} & \mathrm{OMe} & \mathrm{H} & \mathrm{H}\end{array}$

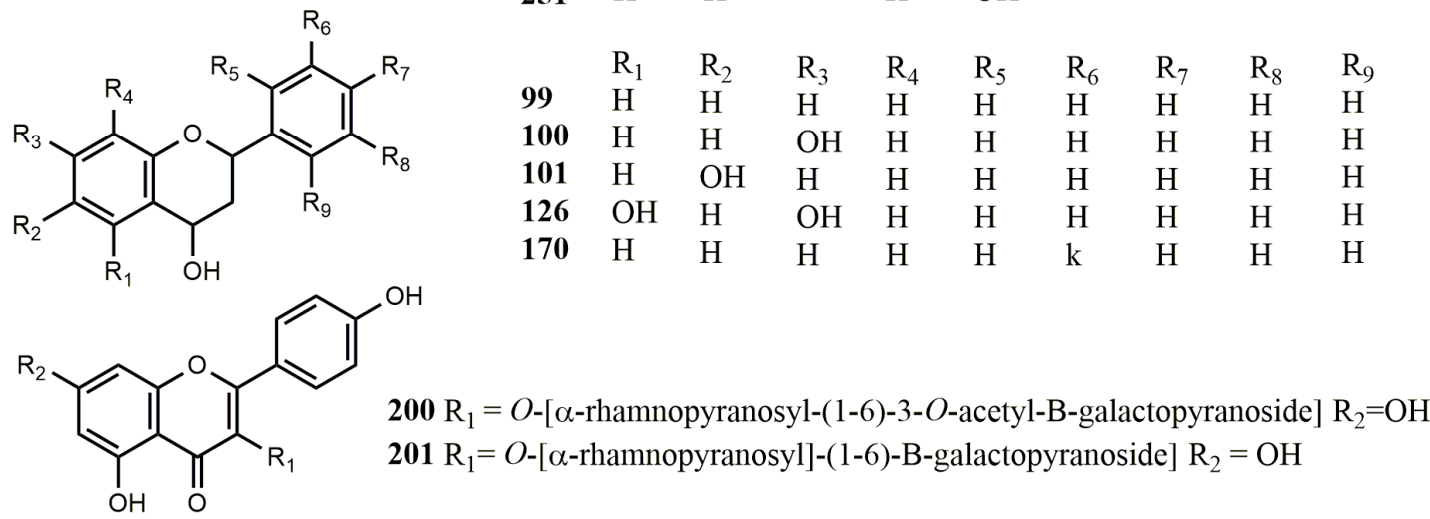

Figure 7. Cont. 
<smiles>[R]c1c([R3])c([R9])c(-c2oc3c([R9])c([R])c([R])c([R])c3c(=O)c2[R6])c([R9])c1[R]</smiles><smiles>C[C@@H]1O[C@H](O[SiH3])[C@@H](O)[C@H](O)[C@H]1O</smiles><smiles>OC[C@H]1O[C@H](O[AlH2])[C@@H](O)[C@H](O)[C@H]1O</smiles><smiles>CO[C@H]1O[C@H](CO)[C@@H](O[AlH])[C@H](O)[C@H]1O</smiles>

$$
\mathrm{l}=
$$<smiles>CNC(C)=O</smiles><smiles>OC[C@H]1O[C@@H](OC[C@H]2O[C@H](OS)[C@@H](O)[C@H](O)[C@H]2O)[C@H](O)[C@@H](O)[C@H]1O</smiles>

$98 \mathrm{H}-\mathrm{H}-\mathrm{H}$

$102 \mathrm{H} \quad \mathrm{OH} \quad \mathrm{H} \quad \mathrm{H}$

$118 \mathrm{H}$ OMe $\mathrm{H} \quad \mathrm{H}$

$119 \mathrm{H} \quad \mathrm{OH} \quad \mathrm{H} \quad \mathrm{H}$

$120 \mathrm{H}$ H OMe $\mathrm{H}$

$121 \mathrm{H} \quad \mathrm{H} \quad \mathrm{OH} \quad \mathrm{H}$

122

136

152

153

154

155

156
157

$158 \mathrm{OH}$

$159 \mathrm{OH} \quad \mathrm{H} \quad \mathrm{H} \quad \mathrm{H}$

$160 \mathrm{H} \quad \mathrm{k} \quad \mathrm{H} \quad \mathrm{H}$

$\begin{array}{lllll}161 & \mathrm{H} & \mathrm{H} & \mathrm{k} & \mathrm{H} \\ 163 & \mathrm{H} & \mathrm{H} & \mathrm{NH}_{2} & \mathrm{H}\end{array}$

$\begin{array}{lllll}164 & \mathrm{H} & \mathrm{H} & 1 & \mathrm{H} \\ \mathbf{1 6 5} & \mathrm{H} & \mathrm{H} & \mathrm{l} & \mathrm{H}\end{array}$

$178 \mathrm{H}$ OMe $\mathrm{H} \quad \mathrm{H}$

$179 \mathrm{H}$ OMe $\mathrm{H} \quad \mathrm{H}$

$180 \mathrm{H}$ OMe $\mathrm{H} \quad \mathrm{H}$

$181 \mathrm{H} \quad \mathrm{H} \quad \mathrm{H} \quad \mathrm{H}$

182

$183 \mathrm{H} \quad \mathrm{H} \quad \mathrm{H} \quad \mathrm{H}$

$184 \mathrm{H} \quad \mathrm{H} \quad \mathrm{H} \quad \mathrm{H}$

$185 \mathrm{H}$

$\begin{array}{lllll}186 & \mathrm{OH} & \mathrm{H} & \mathrm{OH} & \mathrm{H} \\ 187 & \mathrm{OH} & \mathrm{H} & \mathrm{OH} & \mathrm{H}\end{array}$

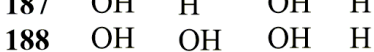

$189 \mathrm{OH} \quad \mathrm{k} \quad \mathrm{OH} \quad \mathrm{H}$

$194 \mathrm{OH} \quad \mathrm{H} \quad \mathrm{OH} \quad \mathrm{H}$

195

196

197

198

202

203

205

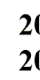

$208 \mathrm{H}-\mathrm{H}-\mathrm{H}$

$209 \mathrm{H} \quad \mathrm{H} \quad \mathrm{H} \quad \mathrm{H}$

$210 \mathrm{H} \quad \mathrm{H} \quad \mathrm{H} \quad \mathrm{k}$

$211 \mathrm{H} \quad \mathrm{H} \quad \mathrm{H} \quad \mathrm{H}$

$212 \mathrm{H} \quad \mathrm{H} \quad \mathrm{H} \quad \mathrm{H}$

213 H $\mathrm{H}$ H

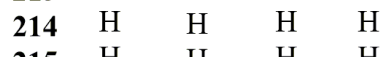

$\begin{array}{lllll}\mathbf{2 1 5} & \mathrm{H} & \mathrm{H} & \mathrm{H} & \mathrm{H} \\ \mathbf{2 1 6} & \mathrm{H} & \mathrm{H} & \mathrm{H} & \mathrm{H}\end{array}$

$217 \mathrm{H} \quad \mathrm{H} \quad \mathrm{H} \quad \mathrm{H}$

$218 \mathrm{H} \quad \mathrm{H} \quad \mathrm{H} \quad \mathrm{H}$

$219 \mathrm{H} \quad \mathrm{H} \quad \mathrm{H} \quad \mathrm{H}$

$220 \mathrm{H} \quad \mathrm{H} \quad \mathrm{H} \quad \mathrm{H}$

$221 \mathrm{H} \quad \mathrm{H} \quad \mathrm{H} \quad \mathrm{H}$

222

223

224

225

$\begin{array}{llll}\mathrm{H} & \mathrm{H} & \mathrm{H} & \mathrm{H}\end{array}$

$\begin{array}{llll}\mathrm{H} & \mathrm{H} & \mathrm{H} & \mathrm{H}\end{array}$

$\begin{array}{llll}\mathrm{H} & \mathrm{k} & \mathrm{H} & \mathrm{H} \\ \mathrm{H} & \mathrm{OH} & \mathrm{H} & \mathrm{H}\end{array}$

\begin{tabular}{|c|c|c|c|c|c|}
\hline $\mathrm{R}_{5}$ & $\mathrm{R}_{6}$ & $\mathrm{R}_{7}$ & $\mathrm{R}_{8}$ & $\mathrm{R}_{9}$ & $\mathrm{R}_{10}$ \\
\hline $\mathrm{H}$ & $\mathrm{H}$ & $\mathrm{H}$ & $\mathrm{H}$ & $\mathrm{H}$ & $\mathrm{H}$ \\
\hline $\mathrm{H}$ & $\mathrm{H}$ & $\mathrm{H}$ & $\mathrm{H}$ & $\mathrm{H}$ & $\mathrm{OH}$ \\
\hline $\mathrm{H}$ & $\mathrm{H}$ & $\mathrm{H}$ & H. & $\mathrm{H}$ & $\mathrm{H}$ \\
\hline $\mathrm{H}$ & $\mathrm{H}$ & $\mathrm{H}$ & $\mathrm{H}$ & $\mathrm{H}$ & $\mathrm{H}$ \\
\hline $\mathrm{H}$ & $\mathrm{H}$ & $\mathrm{OH}$ & $\mathrm{H}$ & $\mathrm{H}$ & $\mathrm{H}$ \\
\hline $\mathrm{H}$ & $\mathrm{H}$ & $\mathrm{H}$ & $\mathrm{H}$ & $\mathrm{H}$ & $\mathrm{H}$ \\
\hline $\mathrm{H}$ & $\mathrm{H}$ & $\mathrm{H}$ & $\mathrm{H}$ & $\mathrm{H}$ & $\mathrm{H}$ \\
\hline $\mathrm{H}$ & $\mathrm{H}$ & $\mathrm{OH}$ & $\mathrm{H}$ & $\mathrm{H}$ & $\mathrm{H}$ \\
\hline $\mathrm{H}$ & $\mathrm{H}$ & $\mathrm{OH}$ & $\mathrm{H}$ & $\mathrm{H}$ & $\mathrm{H}$ \\
\hline $\mathrm{H}$ & $\mathrm{H}$ & $\mathrm{OH}$ & $\mathrm{OMe}$ & $\mathrm{H}$ & $\mathrm{H}$ \\
\hline $\mathrm{H}$ & $\mathrm{H}$ & $\mathrm{H}$ & $\mathrm{H}$ & $\mathrm{H}$ & $\mathrm{H}$ \\
\hline $\mathrm{H}$ & $\mathrm{H}$ & $\mathrm{H}$ & $\mathrm{H}$ & $\mathrm{H}$ & $\mathrm{H}$ \\
\hline $\mathrm{H}$ & $\mathrm{H}$ & $\mathrm{k}$ & $\mathrm{H}$ & $\mathrm{H}$ & $\mathrm{H}$ \\
\hline $\mathrm{k}$ & $\mathrm{H}$ & $\mathrm{H}$ & $\mathrm{H}$ & $\mathrm{H}$ & $\mathrm{H}$ \\
\hline $\mathrm{H}$ & $\mathrm{H}$ & $\mathrm{k}$ & $\mathrm{H}$ & $\mathrm{H}$ & $\mathrm{H}$ \\
\hline $\mathrm{H}$ & $\mathrm{OH}$ & $\mathrm{k}$ & $\mathrm{H}$ & $\mathrm{H}$ & $\mathrm{H}$ \\
\hline $\mathrm{H}$ & $\mathrm{H}$ & $\mathrm{H}$ & $\mathrm{H}$ & $\mathrm{H}$ & $\mathrm{H}$ \\
\hline $\mathrm{H}$ & $\mathrm{H}$ & $\mathrm{k}$ & $\mathrm{H}$ & $\mathrm{H}$ & $\mathrm{H}$ \\
\hline $\mathrm{H}$ & $\mathrm{H}$ & $\mathrm{H}$ & $\mathrm{H}$ & $\mathrm{H}$ & $\mathrm{H}$ \\
\hline $\mathrm{H}$ & $\mathrm{H}$ & $\mathrm{H}$ & $\mathrm{H}$ & $\mathrm{H}$ & $\mathrm{H}$ \\
\hline $\mathrm{H}$ & $\mathrm{H}$ & $\mathrm{H}$ & $\mathrm{H}$ & $\mathrm{H}$ & $\mathrm{H}$ \\
\hline $\mathrm{H}$ & $\mathrm{H}$ & $\mathrm{H}$ & $\mathrm{H}$ & $\mathrm{H}$ & $\mathrm{H}$ \\
\hline $\mathrm{H}$ & $\mathrm{H}$ & $\mathrm{OH}$ & $\mathrm{H}$ & $\mathrm{H}$ & $\mathrm{H}$ \\
\hline $\mathrm{H}$ & $\mathrm{k}$ & $\mathrm{H}$ & $\mathrm{H}$ & $\mathrm{H}$ & $\mathrm{H}$ \\
\hline $\mathrm{H}$ & $\mathrm{H}$ & $\mathrm{k}$ & $\mathrm{H}$ & $\mathrm{H}$ & $\mathrm{H}$ \\
\hline $\mathrm{H}$ & $\mathrm{OH}$ & $\mathrm{k}$ & $\mathrm{H}$ & $\mathrm{H}$ & $\mathrm{H}$ \\
\hline $\mathrm{H}$ & $\mathrm{H}$ & $\mathrm{H}$ & $\mathrm{H}$ & $\mathrm{H}$ & $\mathrm{k}$ \\
\hline $\mathrm{H}$ & $\mathrm{H}$ & $\mathrm{H}$ & $\mathrm{H}$ & $\mathrm{H}$ & $\mathrm{h}$ \\
\hline $\mathrm{H}$ & $\mathrm{H}$ & $\mathrm{H}$ & $\mathrm{H}$ & $\mathrm{H}$ & $\mathrm{m}$ \\
\hline $\mathrm{H}$ & $\mathrm{H}$ & $\mathrm{H}$ & $\mathrm{H}$ & $\mathrm{H}$ & $\mathrm{OMe}$ \\
\hline $\mathrm{H}$ & $\mathrm{H}$ & $\mathrm{k}$ & $\mathrm{H}$ & $\mathrm{H}$ & $\mathrm{OMe}$ \\
\hline $\mathrm{H}$ & $\mathrm{OH}$ & $\mathrm{OH}$ & $\mathrm{H}$ & $\mathrm{H}$ & $\mathrm{OH}$ \\
\hline $\mathrm{H}$ & $\mathrm{OH}$ & $\mathrm{OH}$ & $\mathrm{H}$ & $\mathrm{H}$ & $\mathrm{k}$ \\
\hline $\mathrm{H}$ & $\mathrm{H}$ & $\mathrm{H}$ & $\mathrm{H}$ & $\mathrm{H}$ & $\mathrm{H}$ \\
\hline $\mathrm{H}$ & $\mathrm{H}$ & $\mathrm{H}$ & $\mathrm{H}$ & $\mathrm{H}$ & $\mathrm{H}$ \\
\hline $\mathrm{H}$ & $\mathrm{OH}$ & $\mathrm{OH}$ & $\mathrm{H}$ & $\mathrm{H}$ & $\mathrm{H}$ \\
\hline $\mathrm{H}$ & $\mathrm{k}$ & $\mathrm{OH}$ & $\mathrm{H}$ & $\mathrm{H}$ & $\mathrm{H}$ \\
\hline $\mathrm{H}$ & $\mathrm{OH}$ & $\mathrm{k}$ & $\mathrm{H}$ & $\mathrm{H}$ & $\mathrm{H}$ \\
\hline $\mathrm{H}$ & $\mathrm{OH}$ & $\mathrm{OMe}$ & $\mathrm{H}$ & $\mathrm{H}$ & $\mathrm{H}$ \\
\hline $\mathrm{H}$ & $\mathrm{k}$ & $\mathrm{OMe}$ & $\mathrm{H}$ & $\mathrm{H}$ & $\mathrm{H}$ \\
\hline $\mathrm{H}$ & $\mathrm{H}$ & $\mathrm{OH}$ & $\mathrm{H}$ & $\mathrm{H}$ & $\mathrm{OH}$ \\
\hline $\mathrm{H}$ & $\mathrm{H}$ & $\mathrm{OH}$ & $\mathrm{H}$ & $\mathrm{H}$ & $\mathrm{OH}$ \\
\hline $\mathrm{H}$ & $\mathrm{H}$ & $\mathrm{H}$ & $\mathrm{H}$ & $\mathrm{OMe}$ & $\mathrm{H}$ \\
\hline $\mathrm{H}$ & $\mathrm{H}$ & $\mathrm{H}$ & $\mathrm{OMe}$ & $\mathrm{H}$ & $\mathrm{H}$ \\
\hline $\mathrm{H}$ & $\mathrm{H}$ & $\mathrm{OMe}$ & $\mathrm{H}$ & $\mathrm{H}$ & $\mathrm{H}$ \\
\hline $\mathrm{H}$ & $\mathrm{OMe}$ & $\mathrm{H}$ & $\mathrm{H}$ & $\mathrm{OMe}$ & $\mathrm{H}$ \\
\hline $\mathrm{H}$ & $\mathrm{OMe}$ & $\mathrm{OMe}$ & $\mathrm{OMe}$ & $\mathrm{H}$ & $\mathrm{H}$ \\
\hline $\mathrm{H}$ & $\mathrm{H}$ & $\mathrm{H}$ & $\mathrm{H}$ & $\mathrm{k}$ & $\mathrm{H}$ \\
\hline $\mathrm{H}$ & $\mathrm{H}$ & $\mathrm{H}$ & $\mathrm{H}$ & $\mathrm{OMe}$ & $\mathrm{H}$ \\
\hline $\mathrm{H}$ & $\mathrm{k}$ & $\mathrm{H}$ & $\mathrm{H}$ & $\mathrm{OMe}$ & $\mathrm{H}$ \\
\hline OMe & $\mathrm{H}$ & $\mathrm{H}$ & $\mathrm{H}$ & $\mathrm{H}$ & $\mathrm{k}$ \\
\hline $\mathrm{H}$ & $\mathrm{H}$ & $\mathrm{H}$ & $\mathrm{H}$ & $\mathrm{OH}$ & $\mathrm{H}$ \\
\hline $\mathrm{H}$ & $\mathrm{H}$ & $\mathrm{H}$ & $\mathrm{OH}$ & $\mathrm{H}$ & $\mathrm{H}$ \\
\hline H & H & $\mathrm{H}$ & $\mathrm{k}$ & $\mathrm{H}$ & H \\
\hline $\mathrm{H}$ & $\mathrm{H}$ & $\mathrm{OH}$ & $\mathrm{H}$ & $\mathrm{H}$ & $\mathrm{H}$ \\
\hline $\mathrm{H}$ & $\mathrm{H}$ & $\mathrm{k}$ & $\mathrm{H}$ & $\mathrm{H}$ & $\mathrm{H}$ \\
\hline $\mathrm{H}$ & $\mathrm{OMe}$ & $\mathrm{H}$ & $\mathrm{H}$ & $\mathrm{k}$ & $\mathrm{H}$ \\
\hline $\mathrm{H}$ & $\mathrm{OMe}$ & $\mathrm{k}$ & $\mathrm{H}$ & $\mathrm{OMe}$ & $\mathrm{H}$ \\
\hline $\mathrm{H}$ & $\mathrm{OH}$ & $\mathrm{H}$ & $\mathrm{H}$ & $\mathrm{OMe}$ & $\mathrm{H}$ \\
\hline $\mathrm{H}$ & $\mathrm{OMe}$ & $\mathrm{OH}$ & $\mathrm{H}$ & OMe & $\mathrm{H}$ \\
\hline $\mathrm{H}$ & $\mathrm{OMe}$ & OMe & $\mathrm{k}$ & $\mathrm{H}$ & $\mathrm{H}$ \\
\hline $\mathrm{H}$ & $\mathrm{OMe}$ & $\mathrm{k}$ & OMe & $\mathrm{H}$ & $\mathrm{H}$ \\
\hline $\mathrm{H}$ & OMe & $\mathrm{OMe}$ & $\mathrm{OMe}$ & $\mathrm{H}$ & $\mathrm{H}$ \\
\hline $\mathrm{H}$ & $\mathrm{OMe}$ & $\mathrm{OMe}$ & $\mathrm{k}$ & $\mathrm{H}$ & $\mathrm{H}$ \\
\hline
\end{tabular}

Figure 7. Cont. 

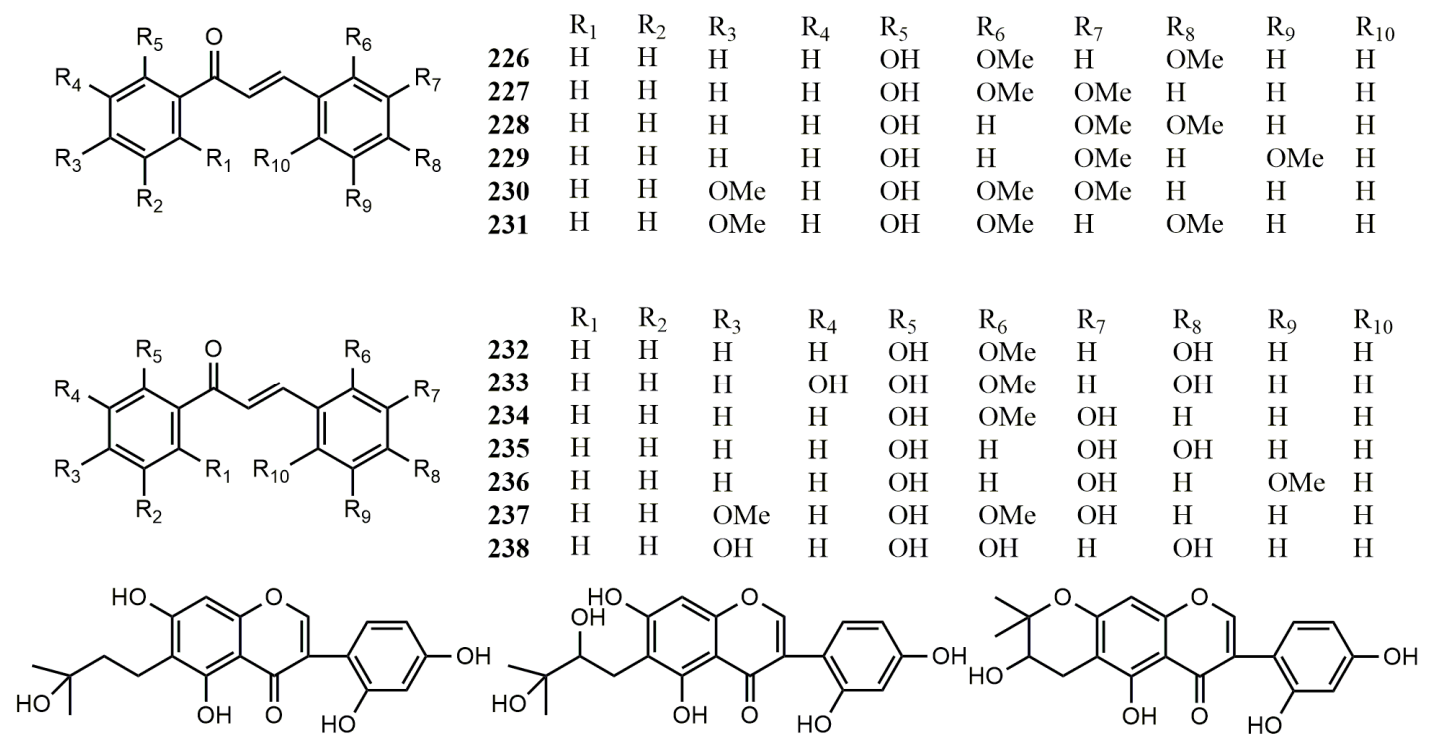

89
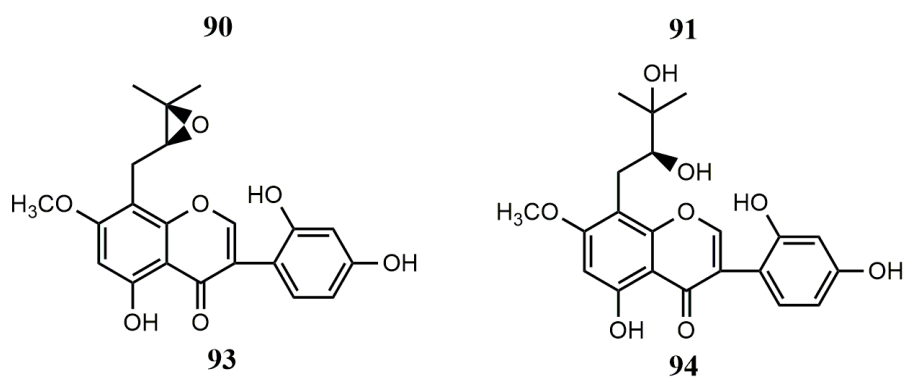<smiles>CC(C)=CCc1c(O)cc2occ(-c3ccc(O)cc3)c(=O)c2c1O</smiles>

103<smiles>CC(C)(O)C1Cc2c(cc3occ(-c4ccc(O)cc4)c(=O)c3c2O)O1</smiles>

104<smiles>CC1(C)Oc2cc3occ(-c4ccc(O)cc4)c(=O)c3c(O)c2CC1O</smiles>

105<smiles>CC(C)(O)CCc1c(O)cc2occ(-c3ccc(O)cc3)c(=O)c2c1O</smiles>

107

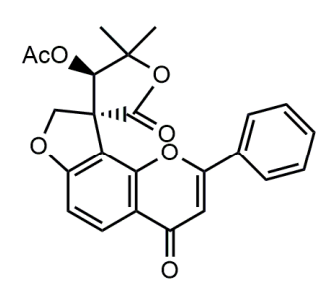

110<smiles>CC(=O)O[C@@H]1C(=O)Oc2ccc3c(=O)cc(-c4ccccc4)oc3c21</smiles>

111<smiles>[R]c1ccc(CCC(=O)c2cc([R6])ccc2O)cc1</smiles>

$112 \mathrm{R}_{1}=\mathrm{OH} \mathrm{R} \mathrm{R}_{2}=\mathrm{H}$

$113 \mathrm{R}_{1}=\mathrm{H} \quad \mathrm{R}_{2}=\mathrm{H}$

$115 \mathrm{R}_{1}=\mathrm{H} \quad \mathrm{R}_{2}=\mathrm{OH}$<smiles>CC(C)=CCc1c(O)cc(O)c2c1O[C@H](c1ccc(O)cc1)CC2=O</smiles>

117<smiles>COc1cc(O)c(CC=C(C)C)c(O)c1C(=O)/C=C/c1ccc(O)cc1</smiles>

Figure 7. Cont. 


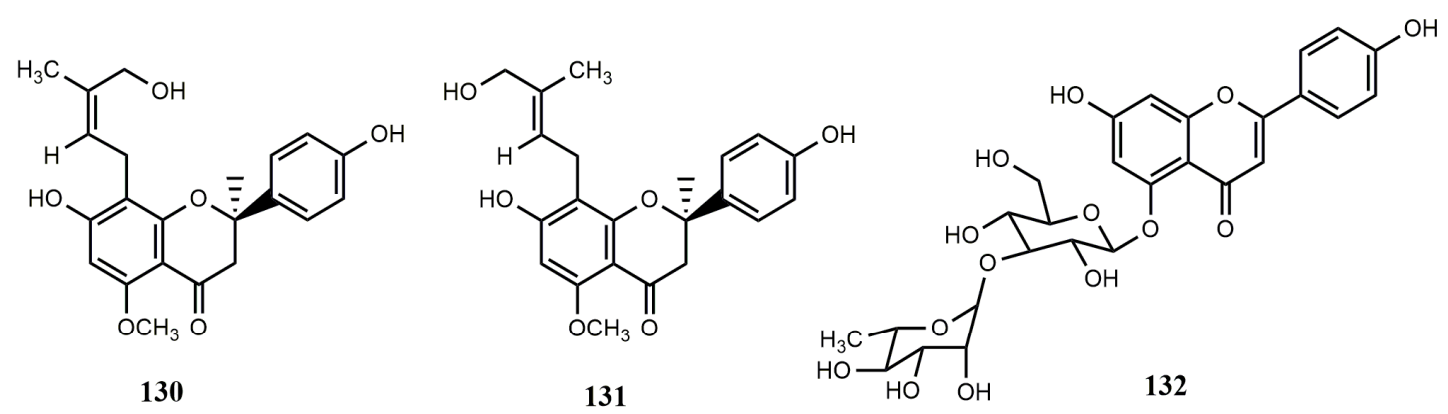<smiles></smiles><smiles>COc1cc2c(cc1CC=C(C)C)C(=O)CC(c1ccc(O)cc1)O2</smiles>

143<smiles>CC(C)=CCc1cc2c(cc1O)OC(c1ccc(O)cc1)CC2=O</smiles>

144<smiles>CC(C)=CCc1c(O)ccc(C(=O)/C=C/c2ccc(O)cc2)c1O</smiles>

145<smiles>COc1cc2c(cc1C/C=C(\C)CO)C(=O)CC(c1ccc(O)cc1)O2</smiles>

146

147

148<smiles>CC(C)=CCc1c(OC2OC(C(O)O)C(O)C(O)C(O)C2O)ccc(C(=O)/C=C/c2ccc(O)cc2)c1O</smiles>

Figure 7. Biotransformation of isoflavones and flavonoids 83-251 by fungi. 
<smiles>[R]c1c([R])c([R])c(-c2coc3c([R])c([R])c([R])c([R])c3c2=O)c([R])c1[R]</smiles>

$\begin{array}{llllll} & \mathrm{R}_{1} & \mathrm{R}_{2} & \mathrm{R}_{3} & \mathrm{R}_{4} & \mathrm{R}_{5} \\ \mathbf{2 5 2} & \mathrm{OH} & \mathrm{Cl} & \mathrm{OH} & \mathrm{Cl} & \mathrm{H} \\ \mathbf{2 6 3} & \mathrm{OH} & \mathrm{H} & \mathrm{O} & \mathrm{H} & \mathrm{H} \\ \mathbf{2 6 7} & \mathrm{H} & \mathrm{OMe} & \mathrm{OH} & \mathrm{H} & \mathrm{H} \\ \mathbf{2 7 8} & \text { OMe } & \mathrm{H} & \mathrm{OH} & \mathrm{H} & \mathrm{H} \\ \mathbf{2 7 9} & \text { OMe } & \mathrm{H} & \text { OMe } & \text { OMe } & \mathrm{H} \\ \mathbf{2 8 0} & \text { OMe } & \mathrm{H} & \text { OH } & \text { OMe } & \mathrm{H}\end{array}$

$\begin{array}{llll}\mathrm{R}_{6} & \mathrm{R}_{7} & \mathrm{R}_{8} & \mathrm{R}_{9} \\ \mathrm{H} & \mathrm{OH} & \mathrm{H} & \mathrm{H} \\ \mathrm{H} & \mathrm{OH} & \mathrm{H} & \mathrm{H} \\ \mathrm{OH} & \mathrm{OH} & \mathrm{H} & \mathrm{H} \\ \mathrm{H} & \mathrm{OH} & \mathrm{H} & \mathrm{H} \\ \mathrm{H} & \mathrm{OH} & \mathrm{H} & \mathrm{H} \\ \mathrm{H} & \mathrm{OH} & \mathrm{H} & \mathrm{H}\end{array}$<smiles>[R]c1c([R])c([R])c(-c2oc3c([R])c([R])c([R])c([R])c3c(=O)c2[R])c([R])c1[R]</smiles><smiles>O=C(O)[C@H]1O[C@H](O[AlH2])[C@@H](O)[C@H](O)[C@@H]1O</smiles><smiles>C/C([O-])=C\CC/C(C)=C/CO[Te]</smiles><smiles>CC(C=[PH2+])=CCCC(C)=CCS</smiles>

253 $\begin{array}{llll}\mathrm{H} & \mathrm{H} & \mathrm{R}_{3} & \mathrm{R}_{4} \\ \mathrm{H} & \mathrm{OH} & \mathrm{H} & \mathrm{H}\end{array}$ $\begin{array}{lllll}\mathrm{H} & \mathrm{H} & \mathrm{H} & \mathrm{H} & \mathrm{H}\end{array}$ $\begin{array}{llll}\mathrm{H} & \mathrm{OH} & \mathrm{H} & \mathrm{H}\end{array}$ $\mathrm{OH} \quad \mathrm{H} \quad \mathrm{O} \quad \mathrm{H}$ $\begin{array}{lllll} & \mathrm{O} & \mathrm{OH} & \mathrm{H} & \mathrm{H}\end{array}$ $\mathrm{H} \quad \mathrm{H}$ $\mathrm{H} \quad \mathrm{H}$ $\mathrm{OH} \quad \mathrm{H}$ $\mathrm{OH} \quad \mathrm{H}$

$\mathrm{OH}$ $\mathrm{OH} \quad \mathrm{H}$ $\mathrm{OH} \quad \mathrm{H}$ $\mathrm{H} \quad \mathrm{H}$ $\mathrm{OH} \quad \mathrm{H}$ $\mathrm{OH} \quad \mathrm{H}$<smiles>C[C@@H]1O[C@H](OC[C@H]2O[C@H](O[AlH2])[C@@H](O)[C@H](O)[C@H]2O)C(O)[C@H]([18OH])[C@H]1O</smiles><smiles>CCCC</smiles>
254

$\begin{array}{ll}\mathrm{R}_{4} & \mathrm{R}_{5} \\ \mathrm{H} & \mathrm{H} \\ \mathrm{H} & \mathrm{H} \\ \mathrm{H} & \mathrm{H} \\ \mathrm{H} & \mathrm{H} \\ \mathrm{H} & \mathrm{H} \\ \mathrm{p} & \mathrm{H} \\ \mathrm{H} & \mathrm{H} \\ \mathrm{H} & \mathrm{H} \\ \mathrm{H} & \mathrm{H} \\ \mathrm{H} & \mathrm{II} \\ \mathrm{H} & \mathrm{H} \\ \mathrm{OH} & \mathrm{H} \\ \mathrm{H} & \mathrm{H} \\ \mathrm{H} & \mathrm{H}\end{array}$

$\mathrm{R}_{6}$

$\begin{array}{llll}\mathrm{R}_{7} & \mathrm{R}_{8} & \mathrm{R}_{9} & \mathrm{R}_{10}\end{array}$ $\begin{array}{lllll}\mathrm{H} & \mathrm{H} & \mathrm{OH} & \mathrm{OH} & \mathrm{H}\end{array}$

$\mathrm{H}$

$\mathrm{H}$

$\mathrm{H}$

$\mathrm{H}$

$\mathrm{H}$
$\mathrm{H}$

$\mathrm{H}$

$\mathrm{H}$
$\mathrm{H}$

$\mathrm{H}$
$\mathrm{H}$

$\mathrm{H}$
$\mathrm{H}$ $\mathrm{OH}$

$\begin{array}{lll}\mathrm{H} & \mathrm{OH} & \mathrm{H}\end{array}$

$\begin{array}{llll}\mathrm{OH} & \mathrm{H} & \mathrm{H} & \mathrm{H}\end{array}$

$\begin{array}{llll} & \mathrm{H} & \mathrm{H} & \mathrm{H}\end{array}$

$\mathrm{OH} \quad \mathrm{H} \quad \mathrm{H} \quad \mathrm{H}$

$\begin{array}{llll} & \mathrm{H} & \mathrm{H} & \mathrm{H}\end{array}$

$\mathrm{OH} \quad \mathrm{OH} \quad \mathrm{H} \quad \mathrm{q}$

n $\quad$ OH $\quad \mathrm{H} \quad \mathrm{OH}$

$\mathrm{OH} \quad \mathrm{OH} \quad \mathrm{H} \quad \mathrm{OH}$

$\mathrm{OH}$ OII II n

$\mathrm{OH} \quad \mathrm{n} \quad \mathrm{H} \quad \mathrm{OH}$

$\mathrm{H} \quad \mathrm{H} \quad \mathrm{H} \quad \mathrm{H}$

$\begin{array}{llll}\mathrm{OH} & \mathrm{H} & \mathrm{H} & \mathrm{H}\end{array}$

$\begin{array}{llll}\mathrm{OH} & \mathrm{H} & \mathrm{H} & \mathrm{H}\end{array}$<smiles>CC(C)=CCc1c(O)cc2c(c1O)C(=O)CC(c1ccc(O)cc1)O2</smiles><smiles>O=C(O)C1=CC(O)C(O)C(Oc2cc(O)c3c(=O)c(O)c(-c4ccc(O)c(O)c4)oc3c2)O1</smiles><smiles>O=C(CCc1ccc(O)cc1)c1c(O)cc(O)cc1O</smiles>

Figure 8. Biotransformation of isoflavones and flavonoids 252-284 by actinomycetes.

The study on the biotransformation of filamentous fungi found that Trichoderma harzianum NJ01 could convert puerarin (108) to $3^{\prime}$-hydroxypuerarin (109) with a conversion rate of up to $41 \%$ under the optimal conditions. In the DPPH free radical scavenging system, compound 109 was 20 times more active than 108. The solubility of $\mathbf{1 0 9}$ is 1.3 times higher than $\mathbf{1 0 8}$ [71]. Glabratephrin (110) was obtained from Tephrosia purpurea and transformed into pseudosemiglabrin (111) by the culture of 
A. niger. The transformation process is realized by using the open loop and the closed loop of the five-member ring [72].

Flavanone (95) is converted by A. niger MB and Penicillium chermesinum 113, and two flavonoids, 6-hydroxyflavanone (96) and 4-hydroxyflavanone (114), were obtained by hydroxylation, and three dihydrochalcones with hydroxyl groups were formed and identified as $2^{\prime}, 5^{\prime}$-dihydroxydihydrochalcone (112), 2'-hydroxydihydrochalcone (113), and 2',4-dihydroxydihydrochalcone (115), respectively [21].

Prenylated flavonoids are usually sourced from medicinal plants, and because of restrictions on bioavailability and the high extraction costs, it is essential to study their biological origins. Studies on the biotransformation showed that naringenin 8-dimethylallyltransferase expressed by transgenic yeast could convert naringenin (116) into 8-dimethylallylnaringenin (117). This was an example to provide a method for the production of prenylated flavonoids that rarely occur in nature [73].

Biotransformation by A. niger MB using 6- and 7-methoxyflavones as substrates showed that 6-methoxyflavone (118) and 7-methoxyflavone (120) were converted to 6-hydroxyflavone (102) and 7-hydroxyflavone (121) by demethylation at C-6, and then to from 6,4'-dihydroxyflavone (119) and 7,4'-dihydroxyflavone (122) by demethylation at C-6 and hydroxylation at C-4' [74]. The resulting products had a stronger antioxidant activity than the substrates.

The biotransformation of 7-hydroxyflavanone (123) by three Aspergillus strains (A. ochraceus 456, A. niger KB, A. niger 13/5) and one Penicillium strain (P. chermesinum 113) were studied. In P. chermesinum 113, compound 123 was converted to 7 -methoxyflavanone (124) by methylation with a conversion rate of $24 \%$, and to obtain $3^{\prime}, 4^{\prime}$-dihydroxy-7-methoxyflavanone (125) by methylation combined with hydroxylation at C-3 and C-4 positions, with a conversion rate of $19 \%$. In A. ochraceus 456 and A. niger KB, the carbonyl group of compound $\mathbf{1 2 3}$ was reduced to form 2,4-cis-7-hydroxyflavan-4-ol (100) and compound 125, respectively, with each conversion yields of $74 \%$ and $12 \%$. 2,4-trans-5,7-Dihydroxyflavan-4-ol (126) was obtained by $A$. ochraceus transformation using compound 123 as substrate. In A. niger 13/5, substrate 123 was converted to 7-hydroxyflavone (121) by dehydrogenation at C-2 and C-3 positions with a conversion rate of up to $98 \%$. Most of the products were found to have more oxidative activity than the substrate [75].

Xanthohumol (127) was converted as a substrate to obtain (2S)-8-[4"-hydroxy-3"'-methyl-

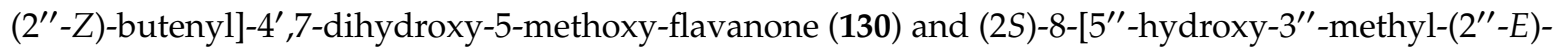
butenyl]-4',7-dihydroxy-5-methoxyflavanone (131) by Cunninghamella echinulata NRRL 3655.

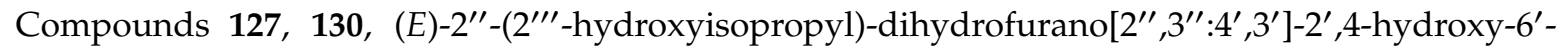
methoxychalcone (128) and (2S)-2"-(2'"'-hydroxyisopropyl)-dihydrofurano[2", $\left.3^{\prime \prime}: 7,8\right]-4^{\prime}$-hydroxy5-methoxyflavanone (129) were obtained from Pichia membranifaciens which showed antimalarial activity against Plasmodium falciparum [76].

Three compounds, apigenin 5-O- $\alpha$-L-rhamnopyranosyl-(1 $\rightarrow 3)-\beta$-D-glucopyranoside (132), 5 -O- $\alpha$-L-rhamnopyranosyl-(1 $\rightarrow 2)-\left(6^{\prime \prime}-O\right.$-acetyl)- $\beta$-D-glucopyranoside (133) and chrysoeriol 5 -O- $\alpha$-L-rhamnopyranosyl- (1 $\rightarrow 4)-(6 "$ "-O-acetyl)- $\beta$-D-glucopyranoside (134) were obtained from the leaves of Cephalotaxus harringtonia, and they were used as substrates for biotransformation by Paraconiothyrium variabile. Compounds 133 and $\mathbf{1 3 4}$ were deglycosylated to form apigenin (135), then compound 134 was converted to chrysoeriol (136) [77].

Biotransformation was performed using isoflavones and $4^{\prime}$-fluoroisoflavones as substrates by A. niger and Cunninghamella elegans. Both fungi rapidly convert isoflavones into several metabolites. They metabolize isoflavones $(40 \mathrm{mg} / \mathrm{L}$ ) with half-lives of 1.6 and 4.2 days, respectively. Twenty-three metabolites were preliminarily identified during the biotransformation of $A$. niger. In the early stage, the main metabolites were mono-hydroxyl and dihydroxyl isoflavones, and after 10 days, the main metabolites were dihydroxyl and trihydroxyl isoflavones. The hydroxylation of isoflavones usually occurs in the B ring. Among them, $3^{\prime}, 4^{\prime}$-dihydroxyl analogues were the most abundant. Methoxy metabolites accumulate slowly during culture. In addition, some glycosides have been detected. However, $4^{\prime}$-fluoroisoflavones were not transformed during culture, indicating that there was regional selective hydroxylation in the initial metabolism of isoflavones [78]. 
$\alpha$-Naphthoflavone (7,8-benzoflavone) (137) and $\beta$-naphthoflavone (5,6-benzoflavone) (139) were transformed by Aspergillus glaucus AM 211, A. niger UPF 724, Penicillium thomi AM 91, Cladosporium avellaneum AM 135, and Verticillium sp. AM 424 via $O$-demethylation and hydroxylation. The substrate $\alpha$-NF was metabolized in seven days to produce $4^{\prime}$-hydroxy- $\alpha$-naphthoflavone (141) and $4^{\prime}$-hydroxy- $\beta$-naphthoflavone (142). The most effective biocatalysts were two strains of AM 211 and AM 424, which converted 137 to 141 with the rates of $43 \%$ and $63 \%$, respectively. Among them, only three strains AM 135, AM 424, and AM 211 can transform $\beta-N F$ (139) into compounds 141 and 142. Besides, $4^{\prime}$-methoxy- $\alpha$-NF (138), a derivative of naphtoflavone, was transformed to compound 141 by A. niger UPF 724 and $P$. thomi AM 91, and the derivative 4 '-methoxy- $\beta$-NF (140) was transformed to compound 142 by $A$. niger UPF 724 [79].

Bavachinin (143) was transformed in the broth of Cunninghamella blakesleeana AS 3.0970 to 4"-hydroxybavachinin (146) and 2",3"-dihydroxybavachinin (147), which were obtained at the yields of $18 \%$ and $7 \%$, respectively, after five days of biotransformation. Biotransformation of bavachin (144) by using C. blakesleeana AS 3.0910 cultures resulted in the formation of three products: (2R,2S)-bavachin 7-O- $\beta$-D-glucopyranoside epimers (148), (2S)-4"-hydroxybavachin (149) and (2S)-5"-hydroxybavachin (150), which were isolated in the yields of $26 \%, 2 \%$, and $5 \%$, after five days of biotransformation. In addition, C. blakesleeana AS 3.0910 transformed isobavachalcone (145) to isobavachalcone $4-O-\beta$-D-glucopyranoside (151), which was obtained in $23 \%$ yields, after 5 days of biotransformation [80]. 6-Methylflavone (152) was transformed in the broth of entomopathogenic fungus Isaria fumosorosea $\mathrm{KCH}$ J2 to 6-methylflavone 8-O- $\beta$-D-(4"-O-methyl)-glucopyranoside (153) and 6-methylflavone $4^{\prime}-O-\beta$-D-(4"-O-methyl)-glucopyranoside (154), which were obtained in $60.5 \%$ and $39.5 \%$ yields, respectively, after 7 days of biotransformation [81].

Isaria filamentous fungi are often used as effective biocatalysts for the transformation of flavonoids. Flavone (97) was metabolized by I. fumosorosea $\mathrm{KCH} \mathrm{J} 2$ in 5 days to produce flavone 2'-O- $\beta$-D-(4"'-O-methyl)-glucopyranoside (155), flavone 4'-O- $\beta$-D-(4'-O-methyl)-glucopyranoside (156) and $3^{\prime}$-hydroxyflavone $4^{\prime}-O-\beta$-D-(4"-O-methyl)-glucopyranoside (157) with the yields of $5.5 \%, 14 \%$ and $4 \%$, respectively. Compound $\mathbf{1 5 6}$ was also obtained when using Isaria farinosa J1.6 as biocatalyst. Besides, 5-hydroxyflavone (158) was converted into 5-hydroxyflavone 4'-O- $\beta$-D-(4"-O-methyl)-glucopyranoside (159) with $29 \%$ yield after 7 days in the culture of strain $\mathrm{KCH} \mathrm{J2}$. Compound 159 was also obtained under the biocatalysis of I. farinosa J1.4. Compound 102, 6-hydroxyflavone, was metabolized by strain $\mathrm{KCH} \mathrm{J2}$ in 5 days to produce 6-O- $\beta$-D-(4"-O-methyl)- glucopyranoside (160) with $13 \%$ yield. Compound 160 was obtained when I. farinosa KW1.2 was used as a biocatalyst. 7-Hydroxyflavone (121) was metabolized by strain $\mathrm{KCH} \mathrm{J2}$ in 7 days to produce 7-O- $\beta$-D-(4"-O-methyl)-glucopyranoside (161) with $16 \%$ yield. Compound $\mathbf{1 6 1}$ was additionally obtained when both of the strains J1.4 and J1.6 were used as a biocatalyst. Daidzein (1) was metabolized by strain $\mathrm{KCH} \mathrm{J2} \mathrm{in} 7$ days to obtain $4^{\prime}$-hydroxyisoflavone 7-O- $\beta$-D-(4"-O-methyl)- glucopyranoside (4"-O-methyldaidzin) (162) with $14.8 \%$ yield. Compound 162 was also obtained when strains KW1.2 and J1.6 were used as biocatalyst. 7-Aminoflavone (163) was metabolized by strain $\mathrm{KCH} \mathrm{J2}$ in 7 days to obtain 7-acetamidoflavone (164) with $10 \%$ yield and $4^{\prime}$-hydroxy-7-acetamidoflavone (165) with 3\% yield [82].

There is a similar report about I. fumosorosea $\mathrm{KCH}$ J2. 2'-Methoxyflavanone (166) was transformed in the broth of the strain $\mathrm{KCH} \mathrm{J2}$ to $2^{\prime}$-methoxyflavanone $5^{\prime}$-O- $\beta$-D-(4"-O-methyl)-glucopyranoside (167) with $18.6 \%$ yield and flavan-4-ol 2 '-O- $\beta$-D-(4"-O-methyl)-glucopyranoside (168) with $24.9 \%$ yield after 11 days of biotransformation. $3^{\prime}$-Methoxyflavanone (169) was metabolized by strain $\mathrm{KCH} \mathrm{J2}$ in 7 days to produce flavan-4-ol $3^{\prime}-O-\beta-\mathrm{D}-\left(4^{\prime \prime}-O-\right.$-methyl)-glucopyranoside (170) with a yield of $15.4 \%$ and $3^{\prime}$-hydroxyflavanone 6-O- $\beta$-D-(4"-O-methyl)-glucopyranoside (171) with a yield of $16 \%$. $4^{\prime}$-Methoxyflavanone (172) was metabolized by strain $\mathrm{KCH} \mathrm{J} 2$ in 7 days to produce $4^{\prime}-O-\beta$-D-(4"-O-methyl)-glucopyranoside (173), $4^{\prime}$-hydroxyflavanone 6-O- $\beta$-D-(4"-O-methyl)-glucopyranoside (174) and $3^{\prime}, 4^{\prime}$-dihydroxyflavanone 6-O- $\beta$-D-(4"-O-methyl)-glucopyranoside (175) with the yields of $11 \%, 20.7 \%$ and $32.8 \%$, respectively. 6-Methoxyflavanone (120) was metabolized by strain $\mathrm{KCH} \mathrm{J} 2$ in 8 days to 
produce 6-methoxyflavanone 4'-O- $\beta$-D-(4"-O-methyl)-glucopyranoside (176), and 3'-hydroxy-6methoxyflavanone-4'-O- $\beta$-D-(4"-O-methyl)-glucopyranoside (177) with the yields of $6 \%$ and $7 \%$, respectively. Compound $\mathbf{1 1 8}$ was metabolized by the strain $\mathrm{KCH}$ J2 in 12 days to produce 6-methoxyflavanone $3^{\prime}-O-\beta$-D-(4"-O-methyl)-glucopyranoside (178), 6-methoxyflavone $4^{\prime}$-O- $\beta$-D-(4"-O-methyl)-glucopyranoside (179), and 3'-hydroxy-6-methoxyflavone $44^{\prime}-O-\beta$-D- $\left(4^{\prime \prime}-O-\right.$ methyl)-glucopyranoside (180) with yields of $2.6 \%, 7.8 \%$, and $7.9 \%$, respectively [83].

When I. fumosorosea $\mathrm{KCH} \mathrm{J2}$ as the biocatalyst, the substrate 3-hydroxyflavone (98) was metabolized in 7 days to produce flavone $3-O-\beta-D-\left(4^{\prime \prime}-O-\right.$ methyl)-glucopyranoside (181), flavone 3-O- $\beta$-D-glucopyranoside (182), and 3-O-[ $\beta$-D-glucopyranosyl- $(1 \rightarrow 6)-\beta$-D-glucopyranosyl]$4^{\prime}$-hydroxyflavone (183) with the conversion rates of $42.5 \%, 5 \%$, and $3.5 \%$, respectively. Compounds 181 and 182 were formed by the biocatalysis of the strains J1.4 and J1.6. Besides, 3-methoxyflavone $4^{\prime}$-O- $\beta$-D-(4"-O-methyl)-glucopyranoside (185) was obtained with $29 \%$ yield from the transformation of 3-methoxyflavone (184) in the culture of strain $\mathrm{KCH} \mathrm{J2}$ for 7 days. Compound $\mathbf{1 8 5}$ was also obtained when strain J1.4 and J1.6 were used as biocatalysts. When the strain $\mathrm{KCH}$ J2 was cultivated for 10 days, $3^{\prime}, 4^{\prime}, 5,7$-tetrahydroxyflavone 3-O- $\beta$-D-(4"-O-methyl)- glucopyranoside (187) and $3^{\prime}, 4^{\prime}, 5,7$-tetrahydroxyflavone 3-O- $\beta$-D-glucopyranoside (isoquercetin) (69) were obtained by biotransformation of 3,3', $4^{\prime}, 5,7$-pentahydroxyflavone (quercetin) (186) with yields of $18.5 \%$ and $12 \%$, respectively. 5,6,7-Trihydroxyflavone (baicalein) (188) was transformed in the broth of the strain $\mathrm{KCH}$ J2 to 5,7-dihydroxyflavone 6-O- $\beta$-D-(4"-O-methyl)-glucopyranoside (189) with yield of 9.7\% after 14 days [84].

Glycosylation is an effective means to regulate the solubility, stability, bioavailability, and bioactivity of natural product drugs. Several important flavonoid aglycones were studied for their glycosylated transformation by microorganisms. Naringenin (116) was glycosylated to form 4',5-dihydroxyflavanone 7-O- $\beta$-D-(4-O-methyl) glucopyranoside (190), 5,7-dihydroxyflavanone 4'-O- $\beta$-D-(4-O-methyl) glucopyranoside (191), 5,7-dihydroxyflavanone 4'-O- $\beta$-D-glucopyranoside (192) and 5-hydroxyflavanone $4^{\prime}, 7$-di-O- $\beta$-D-(4-O-methyl) glucopyranoside (193) by I. fumosorosea ACCC 37814. Luteolin (194) was transformed to $4^{\prime}, 5,7$-trihydroxyflavone $3^{\prime}-O-\beta$-D-(4-O-methyl) glucopyranoside (195) and $3^{\prime}, 5,7$ - trihydroxyflavone $4^{\prime}-O-\beta$-D-(4-O-methyl) glucopyranoside (196) by the strain ACCC 37814 via (4-O-methyl)-glucosyl substituted. Diosmetin (197) was transformed to 5,7-dihydroxy-4'-methoxyflavone $3^{\prime}-O-\beta$-D-(4-O-methyl) glucopyranoside (198) by the strain ACCC 37814 via 4-O-methyl-glucosyl substituted. Formononetin (6) was glycosylated to generate 4'-methoxyisoflavone 7-O- $\beta$-D-(4-O-methyl) glucopyranoside (199) by the strain ACCC 37814 [85].

The host plant-derived flavonoid, kaempferol-O-glycoside (203), was glycosylated and acetylated to produce kaempferol 3-O-[ $\alpha$-rhamnopyranosyl-( $1 \rightarrow 6)-\beta$-galactopyranoside] (201), flavonol kaempferol (202), and acetylated flavonoid glycoside (200) by the fungal endophyte Epicoccum nigrum. Among them, compound $\mathbf{2 0 0}$ was a new kaempferol-O-diglycoside [86].

$2^{\prime}$-Methoxyflavone (204) was transformed by I. fumosorosea $\mathrm{KCh} \mathrm{J2}$ via demethylation and $4-O$-methylglycosylation. The substrate $2^{\prime}$-methoxyflavone was metabolized in 10 days to produce $2^{\prime}-O-\beta$-D-(4"-O-methylglucopyranosyl)-flavone (209), 8-O- $\beta$-D-(4"-O-methylglucopyranosyl)- $2^{\prime}-$ methoxyflavone (210), $5^{\prime}-O-\beta-\mathrm{D}-\left(4^{\prime \prime}-O-\right.$ methylglucopyranosyl)- $2^{\prime}$-methoxyflavone (211) and 3-O- $\beta$-D-(4"-O-methylglucopyranosyl)-2'-methoxyflavone (212). Compound 204 was transformed to 2'-hydroxyflavone (213) by Beauveria bassiana $\mathrm{KCh} \mathrm{J1}$. Similar to the biotransformation of compound 204, 3'-methoxyflavone (205) was converted to $3^{\prime}$-hydroxyflavone (214) by the strain KCh J1. Apart from this, the strain I. farinosa KCh KW 1.1 biotransformed 3'-methoxyflavone (205)

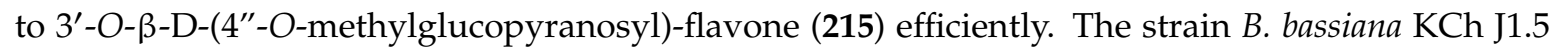
enabled to biotransform $4^{\prime}$-methoxyflavone (206) to $4^{\prime}$-hydroxyflavone (216). Besides, compound 206 was transformed to $4^{\prime}-O-\beta-D-\left(4^{\prime \prime}-O-\right.$-methylglucopyranosyl)-flavone (217) by B. bassiana KCh J1.5. $2^{\prime}, 5^{\prime}$-Dimethoxyflavone (207) was transformed to 211, 2'-O- $\beta$-D-(4"'-O-methylglucopyranosyl)- $5^{\prime}$ methoxyflavone (218) and $4^{\prime}$-O- $\beta$-D-( $4^{\prime \prime}-O$-methylglucopyranosyl)- $2^{\prime}, 5^{\prime}$-dimethoxyflavone (219) by B. bassiana KCh J1.5 and I. farinosa KCh KW1.1. Furthermore, 207 was transformed 
to $5^{\prime}$-hydroxy-2'-methoxyflavone (220) and $4^{\prime}$-hydroxy-2', $5^{\prime}$-dimethoxyflavone (221) by the culture of $B$. bassiana KCh J1. $3^{\prime}, 4^{\prime}, 5^{\prime}$-Trimethoxyflavone (208) was transformed to $3^{\prime}-O-\beta$-D-(4"'-O-methylglucopyranosyl)-4', $5^{\prime}$-dimethoxyflavone (222), 4-O-methylglucoside (223), 6-O- $\beta$-D-(4"-O-methylglucopyranosyl)-3' $4^{\prime}, 5^{\prime}$-trimethoxyflavone (224) and $3^{\prime}-O-\beta-D-\left(4^{\prime \prime}-O-\right.$ methylglucopyranosyl)-6-hydroxy-4', $5^{\prime}$-dimethoxyflavone (225) by the culture of $B$. bassiana $\mathrm{KCh}$ J1.5 [87].

Six chalcones, 2", 4"-dimethoxy-2'-hydroxychalcone (226), 2",3"-dimethoxy-2'-hydroxychalcone (227), 3", 4"-dimethoxy-2'-hydroxychalcone (228), 3",5"'-dimethoxy-2'-hydroxychalcone (229), $2^{\prime \prime}, 3^{\prime \prime}, 4^{\prime}$-trimethoxy-2'-hydroxychalcone (230) and 2",4", $4^{\prime}$-trimethoxy-2'-hydroxychalcone (231) can be converted to seven modified chalcones and six flavonoids in the broth of fungus A. niger LSPN001, which passed through two different pathways. Among them, the modified chalcones included 2"'-methoxy-2',5"'-dihydroxychalcone (232), 2"'-methoxy-2', 3',5"'-trihydroxychalcone (233), 2"-methoxy-2',3'-dihydroxychalcone (234), 2',3",4"'-trihydroxychalcone (235), 5"'-methoxy-2',4"dihydroxychalcone (236), $\quad 4^{\prime}, 2^{\prime \prime}$-dimethoxy-2', $3^{\prime \prime}$-dihydroxychalcone $\quad(237), \quad 2^{\prime}, 4^{\prime}, 2^{\prime \prime}, 4^{\prime \prime}$ tetrahydroxychalcone (238). The flavanones chalcones included $2^{\prime}, 4^{\prime}$-dimethoxyflavanone (239), 2'-methoxy-4'-hydroxyflavanone (240), $\quad 4^{\prime}, 8$-dihydroxy-2'-methoxyflavanone (241), $2^{\prime}, 3^{\prime}$-dimethoxyflavanone (242), 3'-hydroxy-2'-methoxyflavanone (243), 3', $4^{\prime}$-dimethoxyflavanone (244), $3^{\prime}, 4^{\prime}$-dihydroxyflavanone (245), 3',5'-dimethoxyflavanone (246), $3^{\prime}$-hydroxy-5'-methoxy flavanone (247), 7,2', $3^{\prime}$-trimethoxyflavanone (248), $3^{\prime}$-hydroxy-7, $2^{\prime}$-dimethoxyflavanone (249), $7,2^{\prime}, 4^{\prime}$-trimethoxyflavanone (250), and $2^{\prime}, 4^{\prime}$-dihydroxy-7-methoxyflavanone (251) [88].

\subsection{Biotransformation of Isoflavones and Flavonoids in Actinomycetes}

$5,7,4^{\prime}$-Trihydroxyisoflavon (2) is present in soybean meal. Fermentation media containing soybean meal could be used to isolate two new isoflavonoids, 8-chlorogenistein (19) and 6,8-dichlorogenistein (252), from Streptomyces griseus. However, the strain could not produce compounds 19 and 252 without soybean meal, and this suggests that the strain could biotransform compound 2 into chlorinated metabolites 19 and 252. These new isoflavonoids were produced through microbial halogenation. When S. griseus was cultivated with radiologically labelled acetate or phenylalanine, no labelled isoflavones were obtained. The result illustrated that isoflavonoids isolated from streptomycetes may have originated from the medium containing plant-derived nutrient components rather than having a microbial biosynthetic origin [63].

A shuffled biphenyl dioxygenase holoenzyme with broad substrate specificity was coded by the bphA1(2072)A2A3A4 gene cluster, and the gene cluster was introduced into Streptomyces lividans. The recombinant $S$. lividans cell could transform flavone (97) and flavanone (95) into $3^{\prime}$-hydroxyflavone (214), 2', 3'-dihydroxyflavone (253), 2' $3^{\prime}$-dihydroxyflavanone (254), $3^{\prime}$-hydroxyflavanone (255), and $2^{\prime}$-hydroxyflavanone (256). In addition, 6-hydroxyflavone (102) and 6-hydroxyflavanone (96) were transformed into $3^{\prime}, 6$-dihydroxyflavone (257) and 2',6-dihydroxyflavanone (258), respectively. Among all the biotransformed compounds, only compounds 253 and 254 exhibited free radical scavenging activity [89].

Two prenyltransferases, NphB and SCO7190, obtained from two Streptomyces sp., namely CL190 and S. coelicolor A3(2), respectively, could synthesize various prenylated compounds from aromatic substrates, including flavonoids. The recombinant NphB and SCO7190 were overproduced in E. coli and purified to homogeneity. Results of the recombinant $\mathrm{NphB}$ catalysis are as follows: 7-O-Geranyl naringenin (259) and 6-geranyl naringenin (260) were synthetized from naringenin (116) and geranyl diphosphate (GPP); 7-O-geranyl apigenin (261) and 6-geranyl apigenin (262) were synthetized from apigenin (135) and GPP; 7-O-geranyl genistein (263) was synthetized from genistein (2) and GPP; 7-O-geranyl daidzein (264) and 8-geranyl daidzein (265) were synthetized from daidzein (1) and GPP. The result of the recombinant SCO7190 catalysis showed that 6-dimethylallyl naringenin (266) was synthetized from naringenin and DMAPP [90]. NphB, a propenyl transferase, can maintain regioselectivity for propenyl transfer in some cases. 
A marine-derived Streptomyces sp. 060524 was able to hydrolyze the glycosidic bond of isoflavone glycosides. Genistein glycosides (33) and daidzein glycosides (34) were converted to genistein (2) and daidzein (1) by $\beta$-glucosidase, respectively. With the catalyzation by hydroxylase, the strain selectively hydroxylated at the $3^{\prime}$-position and transformed compounds $\mathbf{1}$ and $\mathbf{2}$ into $3^{\prime}$-hydroxygenistein (16) and $3^{\prime}$-hydroxydaidzein (9), respectively. In addition, glycitein glycoside (37) was also converted to glycitein (267) by $\beta$-glucosidase. Compounds 2 and 16 possessed excellent cytotoxicity against K562 human chronic leukemia [91].

A series of flavonoids incubating with the strain Streptomyces sp. M52104 could obtain transformational metabolites. Quercetin (63) was converted into quercetin-4'-O- $\beta$-D-glucuronide (269), quercetin-7-O- $\beta$-D-glucuronide (270), quercetin-3-O- $\beta$-D-glucuronide (271), and quercetin-3'-O- $\beta$-D-glucuronide (272) for $65 \mathrm{~h}$ of incubation. Furthermore, rutin (268) was only transformed into compounds 269-271 by incubating $90 \mathrm{~h}$. Both $2 S$-naringenin (273) and $2 S$-naringenin-7-O- $\beta$-D-glucopyranoside (274) were able to convert into naringenin-7-O- $\beta$-Dglucuronide (275) and naringenin- $4^{\prime}-O-\beta$-D-glucuronide (276) by incubating $65 \mathrm{~h}$ [92].

Quercetin (63) incubating with Streptomyces rimosus subsp. rimosus ATCC 10970 was able to be transformed into quercetin-7-O- $\beta-4$ "'-deoxy-hex-4"-enopyranosiduronic acid (277) in $72 \mathrm{~h}$ [93]. Biotransformation of genistein (2) in the culture of Streptomyces sp. MBT76 obtained 4',7-di-hydroxy-5-methoxy-isoflavone (278), 4'-hydroxy-5,7-dimethoxy-isoflavone (88), 4'-hydroxy-5,7,8-trimethoxy-isoflavone (279), and 7,4'-dihydroxy-5,8-dimethoxy-isoflavone (280) [22]. However, methylated isoflavones exhibited lower bioactivity than the unmethylated precursors.

Two flavanones, pinocembrin (54) and naringenin (116), two flavones, chrysin and apigenin, together with a flavonol, kaempferol, were catalyzed by CYP105D7, a cytochrome P450 from Streptomyces avermitilis [94]. Through experiments, CYP105D7 enabled the catalysis of the hydroxylation of 54 and 116. The 3'-position of $\mathbf{1 1 6}$ was hydroxylated by CYP105D7, while the hydroxylated position of $\mathbf{5 4}$ could not be determined. Nonetheless, the retention time detected in HPLC implied that 3-hydoxylation or $4^{\prime}$-hydoxylation did not occur.

A putative O-methyltransferase OMT gene SpOMT7740 was obtained from the sequence information of Streptomyces peucetius ATCC27952. Introducing the gene into E. coli, BL21 was used to detect the substrate promiscuity and perform functional characterization. Flavonoids were added into the fermentation broth of E. coli BL21 for biotransformation. The theoretical and experimental results were as follows: genistein (2), 3-hydroxyflavone (98), luteolin (194), 7,8-dihydroxyflavone (281), and phloretin (282) were successively converted into tri-O-methoxygenistein, 3-O-methoxyflavone, mono-O-methoxyleutolin, 7-hydroxy-8-O-methoxyflavone, and mono-O-methoxyphloretin [95].

Four known isoflavonoids, daidzein (1), genistein (2), daidzein-7-O- $\alpha$-L-rhamnoside (23), and genistein-7-O- $\alpha$-L-rhamnoside (25), were derived from Indonesian actinomycete Streptomyces sp. TPU1401A [32]. Compounds 1 and 2 were able to transform into the 7-O-glycoside derivatives, 23 and 25, respectively, by the strain TPU1401A. Compounds 23 and 25 promoted the strain TPU1401A grown better than compounds $\mathbf{1}$ and $\mathbf{2}$. This phenomenon suggested that the strain TPU1401A transformed isoflavones into isoflavone glycosides to hasten growth.

Studies showed that ring A hydroxyflavones were transformed to the corresponding C $-4^{\prime}$ hydroxylated metabolites; the rate and yields of production were related to the distance between the C-4 carbonyl group and the hydroxyl group in the A ring. During the process of biotransformation in the culture of Streptomyces fulvissimus, 6-hydroxyflavone (102) and 7-hydroxyflavone (121) converted into 6,4'-dihydroxyflavone (119) and 7,4'-dihydroxyflavone (122) with a medium and slow reaction, respectively. 5-Hydroxyflavone (158) was completely transformed to 5, $4^{\prime}$-dihydroxyflavone (283) and $5,3^{\prime}, 4^{\prime}$-dihydroxyflavone (284) for two days with a rapid reaction. These results demonstrated that the highest rate of transformation was observed when the hydroxy group was closest to the carbonyl group [96]. 


\section{Development and Utilization of Flavonoids Synthesis Genes in Streptomyces}

The availability of flavonoids in plants is limited by seasonal or regional variations, low abundance, and instability in the separation of single compounds from complex mixtures. Therefore, expressing plant biosynthesis genes in microorganisms is an effective method to produce these essential metabolites [97]. Streptomyces, one of the most important genera of actinomycetes, is diverse, and more than $50 \%$ of its species can produce various antibiotics and other active compounds of different structural types [98,99]. Enzymes with various catalytic functions in Streptomyces are useful members of an artificial gene cluster constructed in E. coli for the production of plant-specific flavones, including isoflavones and unnatural compounds by fermentation $[100,101]$. Here, we summarize the genes involved in flavonoid synthesis.

The most common method of studying genes that produce flavonoids in Streptomyces is the introduction of gene fragments into E. coli for heterologous expression. In plants, chalcone is a precursor of flavonoid, which can be converted to flavanones by chalcone isomerase, and 4-coumarate: coenzyme A ligase (4CL) is an essential enzyme in the transformation process. The gene fragment that synthesises 4CL has been found in S. coelicolor A3(2) and can be introduced into E. coli for heterogenic expression, which greatly enhances flavonoid production [100,102]. In a study by Kim et al., the SaOMT-2 gene from S. avermitilis MA-4680 was inserted into E. coli Sa-2 for expression, and the enzyme encoded by the SaOMT-2 gene was found to methylate the 7-hydroxyl group of isoflavones and flavonoids. In particular, the biotransformation of E. coli Sa-2 resulted in the production of sakuranetin, an antifungal flavonoid [103]. A gene fragment from S. peucetius ATCC 27952 that encodes an O-methyltransferase (OMT), SpOMT2884, was expressed in E. coli, and the enzyme was found to methylate various flavonoids, with 7,8-dihydroxyflavone being the most favourable substrate. Bioconversion of 7, 8-dihydroxyflavone was as high as $96 \%$ under optimal conditions, and the resulting 7-hydroxy-8-methoxyflavone was purified for in vitro glycosylation to produce glucose. The results indicated that methylation enhances the stability of the substrate, whereas glycation was shown to increase the water solubility of the substrate [104]. The SpOMT7740 gene from S. peucetius ATCC27952 encoding an OMT was cloned into E. coli to methylate different types of flavonoid substrates. The enzyme catalyzes the formation of various natural and non-natural $O$-methoxides [95]. O-Methylated phenylpropanoids are usually found in small quantities in plants. However, studies have indicated that co-culture of E. coli and Streptomyces can activate the expression of the SaOMT2 gene and promote the production of $O$-methylated phenylpropanoids, thus providing a powerful pathway for the production of scarce and valuable $O$-methylated phenylpropanoids [24]. Studies have shown that methylase GerMIII, derived from Streptomyces sp. KCTC 0041BP, can methylate the 4-hydroxyl position of some flavonoids, and quercetin may be the most suitable substrate [105]. The gene encoding oleandomycin glycosyltransferase (OleD GT) from S. antibioticus was introduced into E. coli BL21(DE3) for expression, and the purified recombinant OleD GT catalyzed glycosylation of various flavonoids. Fixation of OleD GT in hybrid nanoparticles of $\mathrm{Fe}_{3} \mathrm{O}_{4} /$ silica/NiO is an effective technique for maintaining its high activity [106]. Not only E. coli but also Streptomyces can be used as engineered strains for heterologous expression of flavonoid-producing genes. In a study, flavonoid and stilbene biosynthesis genes were introduced for heterologous expression in S. venezuelae DHS2001, with the deletion of the native pikromycin polyketide synthesis gene, and the resulting strain produced racemic naringenin and pinocembrin from 4-coumaric acid and cinnamic acid, respectively. The yield of these two flavonoids was considerably increased by codon optimisation, and this is the first report of the phenylpropanoid biosynthesis pathway in the Streptomyces genus [107]. Marin et al. used the heterologous biosynthesis of industrial actinomyces $S$. coelicolor and Streptomyces albus to express the novo biosynthesis genes of three important flavonols, namely myricetin, kaempferol, and quercetin [108]. The shuffled biphenyl dioxygenase holoenzyme encoded by the bphA1 (2072) A2A3A4 gene cluster of S. lividans exhibits a wide range of substrate specificity and hydroxylates several flavonoids and isoflavones; some of the obtained products possess free radical scavenging activity [89]. Prenyltransferase expressed by the prenyltransferase gene from Streptomyces prenyltransferase HypSc (SCO7190) was shown to exhibit 
extensive substrate specificity in the host plant, tomato, and therefore, tomatoes accumulate prenylated flavonoids. This is the first report of prenylated flavonoid accumulation in transgenic plants [109]. Through gene exploration of $S$. clavuligerus, three naringenin-producing genes, namely ncs, ncyP, and tal, were found, all of which are indispensable for the process. This is the first report on the natural production of naringenin in actinomycetes [110]. The queD gene from S. eurythermus ${ }^{\mathrm{T}}$ encodes $^{-}$ quercetinase (QueDHis6), which can convert several flavonols. The biological function of this enzyme was studied and was found to mainly play a role in detoxification rather than in catabolism [111]. The discovery of genes involved in the production of flavonoids by Streptomyces combined with newly developed techniques and increased genetic knowledge to manipulate the biosynthesis pathways is an effective way to achieve large-scale production of the essential flavonoids.

\section{Conclusions}

Flavonoids and isoflavonoids have attracted considerable attention due to their low toxicity and remarkable biological activity. Flavonoid and isoflavone pathways are considered the most characteristic natural product pathways in plants. However, microbial sources of isoflavones and flavonoids have received little attention. This is the first review of the fungal and actinomycete sources of isoflavones and flavonoids. Only 24 new compounds of isoflavones and flavonoids from fungi and actinomycetes have been identified; however, some of these are highly potent bioactive compounds that might be potential drug candidates. Compounds 61 and 58 from Acanthostigmella sp. CL12082 demonstrated potent antifungal activity. Compound $\mathbf{6 1}$ displayed strong antifungal activity against $C$. albicans and $A$. fumigatus, with $\mathrm{IC}_{50}$ values of 0.11 and $0.54 \mu \mathrm{g} / \mathrm{mL}$, respectively. Similarly, compound 58 displayed extremely strong activity against the fungi, C. albicans and A. fumigatus, with $\mathrm{IC}_{50}$ values of 0.035 and $0.10 \mu \mathrm{g} / \mathrm{mL}$, respectively [54]. Compound 76 from Streptomyces sp. (strain G246) showed broad-spectrum antimicrobial activity, and its antimicrobial activity was far superior than that of its positive controls, streptomycin and cycloheximide. Compound $\mathbf{7 6}$ displayed excellent inhibitory activity against $P$. aeruginosa, $S$. enterica, E. faecalis, $S$. aureus, B. cereus, and C. albicans with $\mathrm{IC}_{50}$ values of $16,32,8,1,4$, and $8 \mu \mathrm{g} / \mathrm{mL}$, respectively [60]. The new compounds $77-79$, derived from marine Streptomyces sp. G248, also demonstrated significant broad-spectrum antimicrobial activity against six pathogens, with $\mathrm{IC}_{50}$ values in the range of $1-16 \mu \mathrm{g} / \mathrm{mL}$, and their activities were much higher than those of the positive controls, streptomycin and cycloheximide [61]. To ascertain the therapeutic potential of these compounds, particularly of lavandulylated and halogen atom-bearing flavonoids, further pharmacological, chemical, and toxicological studies are required. Nearly all the isoflavones and flavones from actinomycetes are reported to be produced by Streptomyces sp.; however, two studies have reported the isolation of isoflavones and flavones from actinomycetes, M. aurantiaca 110B and Amycolatopsis sp. [33,43]. The culture medium of $M$. aurantiaca 110B for producing compounds 20, 21, and 71 was investigated, and the results indicated that the compounds could be detected in the culture medium only when soybean cake is added to the medium. The results also indicated that $M$. aurantiaca $110 \mathrm{~B}$ could transform plant daidzein into fucosylated derivatives. Notably, the fermentation medium in which Amycolatopsis sp. produces isoflavones also comprises of soybean meal. Whether these two strains have similar transformation pathways to produce isoflavonoid glycosides requires further investigation.

Because of the low bioavailability of isoflavones and flavonoids, the bioconversion of isoflavones and flavonoids has gradually gained research attention [80]. In this review, we summarised the biotransformation of isoflavones and flavonoids by fungi and actinomycetes. Understanding the biotransformation process helps us elucidate not only the metabolic pathways of these compounds but also their mechanisms of action, toxicity, and pharmacological activity [112-114]. A. niger and Streptomyces sp. are the most adaptable fungi and actinomycetes, respectively, and are the most commonly used biotransformation strains because of their ability to use various isoflavones and flavonoids as substrates for biosynthesis and biotransformation. They usually undergo hydroxylation, methylation, glycosylation, cyclisation, halogenation, and double bond reduction to produce isoflavones 
and flavonoids, which are more beneficial to human health; for instance, methylation enhances substrate stability, hydroxylation enhances antioxidant activity, and glycosylation enhances water solubility. The biotransformation processes in fungi and actinomycetes for producing isoflavone- and flavonoid-derived drugs have gained considerable attention.

Use of bacteria to produce plant-derived compounds is a new metabolic engineering technology that can help us synthesise important drugs and produce bioactive substances. Multiple flavonoid biosynthesis genes of Streptomyces sp. can be heterogeneously expressed in E. coli or optimisation of the gene expression strategy can be employed to obtain heterogenous expression strains. Moreover, transfer of the biosynthesis pathway from the original microorganism to a more compliant alien host may provide an effective platform for the production of desired levels of flavonoids or other novel compounds. To date, only flavonoid synthesis genes have been studied in Streptomyces sp., and no isoflavone synthesis gene has been discovered yet. Future research should focus on elucidating the generation mechanism of isoflavones and flavonoids in microorganisms to promote the utilisation of these compounds in the pharmaceutical field.

Funding: This research was funded by the National Natural Science Foundation of China (No. 81860634), Applied Basic Research Key Project of Yunnan Province (No. 202001BB050029), Major Science and Technology Projects of Yunnan Province (Digitalization, Development and Application of Biotic Resource, 202002AA100007), and Science Research Foundation of Yunnan Province Education Department.

Conflicts of Interest: The authors declare no conflict of interest.

\section{References}

1. Hodgson, J.M.; Croft, K.D.; Puddey, I.B.; Mori, T.A.; Beilin, L.J. Soybean isoflavonoids and their metabolic products inhibit in vitro lipoprotein oxidation in serum. J. Nutr. Biochem. 1996, 7, 664-669. [CrossRef]

2. Andersen, M.; Markham, K.R. Flavonoids, Chemistry, Biochemistry and Applications; CRC Press/Taylor \& Francis: Boca Raton, FL, USA, 2006; pp. 1-1239. ISBN 0-8493-2021-6.

3. Dixon, R.A.; Pasinetti, G.M. Flavonoids and isoflavonoids: From plant biology to agriculture and neuroscience. Plant Physiol. 2010, 154, 453-457. [CrossRef] [PubMed]

4. Akashi, T.; Aoki, T.; Ayabe, S. Cloning and functional expression of a cytochrome P450 cDNA encoding 2-hydroxyisoflavanone synthase involved in biosynthesis of the isoflavonoid skeleton in licorice. Plant Physiol. 1999, 121, 821-828. [CrossRef] [PubMed]

5. Overkamp, S.; Hein, F.; Barz, W. Cloning and characterization of eight cytochrome P450 cDNAs from chickpea (Cicer arietinum L.) cell suspension cultures. Plant Sci. 2000, 155, 101-108. [CrossRef]

6. Steele, C.L.; Gijzen, M.; Qutob, D.; Dixon, R.A. Molecular characterization of the enzyme catalyzing the aryl migration reaction of isoflavonoid biosynthesis in soybean. Arch. Biochem. Biophys. 1999, 367, 146-150. [CrossRef]

7. Reyes-Chilpa, R.; Gómez-Garibay, F.; Moreno-Torres, G.; Jiménez-Estrada, M.; Quiroz-Vásquez, R.I. Flavonoids and Isoflavonoids with antifungal properties from Platymiscium yucatanum heartwood. Holzforschung 1998, 52, 459-462. [CrossRef]

8. Kopustinskiene, D.M.; Jakstas, V.; Savickas, A.; Bernatoniene, J. Flavonoids as Anticancer Agents. Nutrients 2020, 12, 457. [CrossRef]

9. Donnelly, D.M.X.; Boland, G.M. Isoflavonoids and neoflavonoids: Naturally occurring O-heterocycles. Nat. Prod. Rep. 1995, 12, 321-338. [CrossRef]

10. Wang, S.F.; Ridsdill-Smith, T.J.; Ghisalberti, E.L. Role of isoflavonoids in resistance of subterranean clover trifoliates to the redlegged earth mite halotydeus destructor. J. Chem. Ecol. 1998, 24, 2089-2100. [CrossRef]

11. Dixon, R.A.; Steele, C.L. Flavonoids and isoflavonoids-A gold mine for metabolic engineering. Trends Plant Sci. 1999, 4, 394-400. [CrossRef]

12. Dastidar, S.G.; Manna, A.; Kumar, K.A.; Mazumdar, K.; Dutta, N.K.; Chakrabarty, A.N.; Motohashi, N.; Shirataki, Y. Studies on the antibacterial potentiality of isoflavones. Int. J. Antimicrob. Agents 2004, 23, 99-102. [CrossRef] [PubMed]

13. Orhan, D.D.; Ozcelik, B.; Ozgen, S.; Ergun, F. Antibacterial, antifungal, and antiviral activities of some flavonoids. Microbiol. Res. 2010, 165, 496-504. [CrossRef] 
14. Sychrová, A.; Koláriková, I.; Žemlička, M.; Šmejkal, K. Natural compounds with dual antimicrobial and anti-inflammatory effects. Phytochem. Rev. 2020. [CrossRef]

15. Gupta, C.; Prakash, D. Phytonutrients as therapeutic agents. J. Complement. Integr. Med. 2014, 11, 151-169. [CrossRef] [PubMed]

16. Ebada, S.S.; Eze, P.; Okoye, F.B.C.; Esimone, C.O.; Proksch, P. The fungal endophyte Nigrospora oryzae produces quercetin monoglycosides previously known only from plants. ChemistrySelect 2016, 1, 2767-2771. [CrossRef]

17. Kang, H.R.; Lee, D.; Benndorf, R.; Jung, W.H.; Beemelmanns, C.; Kang, K.S.; Kim, K.H. Termisoflavones A-C, isoflavonoid glycosides from termite-associated Streptomyces sp. RB1. J. Nat. Prod. 2016, 79, 3072-3078. [CrossRef] [PubMed]

18. Keller, N.P.; Turner, G.; Bennett, J.W. Fungal secondary metabolism-From biochemistry to genomics. Nat. Rev. Microbiol. 2005, 3, 937-947. [CrossRef]

19. Solecka, J.; Zajko, J.; Postek, M.; Rajnisz, A. Biologically active secondary metabolites from Actinomycetes. Open Life Sci. 2012, 7, 373-390. [CrossRef]

20. Deshpande, B.S.; Ambedkar, S.S.; Shewale, J.G. Biologically active secondary metabolites from Streptomyces. Enzym. Microb. Technol. 1988, 10, 455-473. [CrossRef]

21. Kostrzewa-Suslow, E.; Dmochowska-Gladysz, J.; Bialonska, A.; Ciunik, Z. Microbial transformations of flavanone by Aspergillus niger and Penicillium chermesinum cultures. J. Mol. Catal. B Enzym. 2008, 52-53, 34-39. [CrossRef]

22. Wu, C.; Zhu, H.; van Wezel, G.P.; Choi, Y.H. Metabolomics-guided analysis of isocoumarin production by Streptomyces species MBT76 and biotransformation of flavonoids and phenylpropanoids. Metabolomics 2016, 12, 90. [CrossRef] [PubMed]

23. Moore, B.S.; Hertweck, C.; Hopke, J.N.; Izumikawa, M.; Kalaitzis, J.A.; Nilsen, G.; Noel, J.P. Plant-like biosynthetic pathways in bacteria: From benzoic acid to chalcone. J. Nat. Prod. 2002, 65, 1956-1962. [CrossRef] [PubMed]

24. Cui, H.; Song, M.C.; Ban, Y.H.; Jun, S.Y.; Kwon, A.S.; Lee, J.Y.; Yoon, Y.J. High-yield production of multiple O-methylated phenylpropanoids by the engineered Escherichia coli-Streptomyces cocultivation system. Microb. Cell Fact. 2019, 18, 67. [CrossRef]

25. Dixon, R.A. Isoflavonoids: Biochemistry, molecular biology, and biological functions. Compr. Nat. Prod. Chem. 1999, 1, 773-823. [CrossRef]

26. Adlercreutz, H.; Mazur, W. Phyto-estrogens and western diseases. Ann. Med. 1997, 29, 95-120. [CrossRef]

27. Reynaud, J.; Guilet, D.; Terreux, R.; Lussignol, M.; Walchshofer, N. Isoflavonoids in non-leguminous families: An update. Nat. Prod. Rep. 2005, 22, 504-515. [CrossRef]

28. Mackova, Z.; Koblovska, R.; Lapcik, O. Distribution of isoflavonoids in non-leguminous taxa-an update. Phytochemistry 2006, 67, 849-855. [CrossRef]

29. Lapcik, O. Isoflavonoids in non-leguminous taxa: A rarity or a rule? Phytochemistry 2007, 68, 2910-2916. [CrossRef] [PubMed]

30. Veitch, N.C. Isoflavonoids of the Leguminosae. Nat. Prod. Rep. 2013, 30, 988-1027. [CrossRef]

31. Al-Maharik, N. Isolation of naturally occurring novel isoflavonoids: An update. Nat. Prod. Rep. 2019, 36, 1156-1195. [CrossRef]

32. Wewengkang, D.S.; Yamazaki, H.; Takahashi, M.; Togashi, T.; Rotinsulu, H.; Sumilat, D.A.; Namikoshi, M. Production of an $\alpha$-pyrone metabolite and microbial transformation of isoflavones by an Indonesian Streptomyces sp. J. Asian Nat. Prod. Res. 2020, 22, 754-761. [CrossRef]

33. Zheng, K.X.; Jiang, Y.; Jiang, J.X.; Huang, R.; He, J.; Wu, S.H. A new phthalazinone derivative and a new isoflavonoid glycoside from lichen-associated Amycolatopsis sp. Fitoterapia 2019, 135, 85-89. [CrossRef]

34. Fujita, T.; Funako, T.; Hayashi, H. 8-Hydroxydaidzein, an aldose reductase inhibitor from okara fermented with Aspergillus sp. HK-388. Biosci. Biotechnol. Biochem. 2004, 68, 1588-1590. [CrossRef]

35. Maskey, R.P.; Asolkar, R.N.; Speitling, M.; Hoffman, V.; Grun-Wollny, I.; Fleck, W.F.; Laatsch, H. Flavones and new isoflavone derivatives from microorganisms: Isolation and structure elucidation. Z. Naturforsch. B 2003, 58, 686-691. [CrossRef]

36. Komiyama, K.; Funayama, S.; Anraku, Y.; Mita, A.; Takahashi, Y.; Omura, S.; Shimasaki, H. Isolation of isoflavonoids possessing antioxidant activity from the fermentation broth of Streptomyces sp. J. Antibiot. 1989, 42, 1344-1349. [CrossRef] 
37. Funayama, S.; Anraku, Y.; Mita, A.; Komiyama, K.; Omura, S. Structural study of isoflavonoids possessing antioxidant activity isolated from the fermentation broth of Streptomyces sp. J. Antibiot. 1989, 42, 1350-1355. [CrossRef]

38. Chimura, H.; Sawa, T.; Kumada, Y.; Naganawa, H.; Matsuzaki, M.; Takita, T.; Umezawa, H. New isoflavones, inhibiting catechol-O-methyltransferase, produced by Streptomyces. J. Antibiot. 1975, 28, 619-626. [CrossRef]

39. Umezawa, H.; Tobe, H.; Shibamoto, N.; Nakamura, F.; Nakamura, K.; Matsuzaki, M.; Takeuchi, T. Isolation of isoflavones inhibiting dopa decarboxylase from fungi and Streptomyces. J. Antibiot. 1975, 28, 947-952. [CrossRef] [PubMed]

40. Hazato, T.; Naganawa, H.; Kumagai, M.; Aoyagi, T.; Umezawa, H. $\beta$-galactosidase-inhibiting new isoflavonoids produced by actinomycetes. J. Antibiot. 1979, 32, 217-222. [CrossRef]

41. Lee, S.R.; Song, J.H.; Song, J.H.; Ko, H.J.; Baek, J.Y.; Trinh, T.A.; Beemelmanns, C.; Yamabe, N.; Kim, K.H. Chemical Identification of isoflavonoids from a termite-associated Streptomyces sp. RB1 and their neuroprotective effects in murine hippocampal HT22 cell line. Int J. Mol. Sci. 2018, 19, 2640. [CrossRef]

42. Huang, R.; Ding, Z.G.; Long, Y.F.; Zhao, J.Y.; Li, M.G.; Cui, X.L.; Wen, M.L. A new isoflavone derivative from Streptomyces sp. YIM GS3536. Chem. Nat. Compd. 2013, 48, 966-969. [CrossRef]

43. Wang, R.J.; Zhang, S.Y.; Ye, Y.H.; Yu, Z.; Qi, H.; Zhang, H.; Xue, Z.L.; Wang, J.D.; Wu, M. Three new isoflavonoid glycosides from the mangrove-derived actinomycete Micromonospora aurantiaca 110B. Mar. Drugs 2019, 17, 294. [CrossRef]

44. Tahara, S.; Nakahara, S.; Mizutani, J.; Ingham, J.L. Fungal transformation of the antifungal isoflavone luteone. Agric. Food Chem. 1984, 48, 1471-1477. [CrossRef]

45. Tahara, S.; Nakahara, S.; Mizutani, J.; Ingham, J.L. Fungal metabolism of the prenylated isoflavone licoisoflavone A. Agric. Biol. Chem. 1985, 49, 2605-2612.

46. Ni, M.; Wu, Q.; Wang, G.S.; Liu, Q.Q.; Yu, M.X.; Tang, J. Analysis of metabolic changes in Trichoderma asperellum TJ01 at different fermentation time-points by LC-QQQ-MS. J. Environ. Sci. Health B 2019, 54, $20-26$. [CrossRef]

47. Cheng, M.J.; Wu, M.D.; Cheng, Y.C.; Chen, J.J.; Hsieh, S.Y.; Yuan, G.F.; Su, Y.S. Metabolites Isolated from an endophytic fungus of Annulohypoxylon elevatidiscus. Chem. Nat. Compd. 2015, 51, 67-70. [CrossRef]

48. Cheng, M.J.; Chan, H.Y.; Cheng, Y.C.; Wu, M.D.; Chen, J.J.; Chen, Y.L.; Hsieh, S.Y.; Yuan, G.F.; Su, Y.S. A new pyrrole metabolite from the endophytic fungus of Xylaria papulis. Chem. Nat. Compd. 2015, 51, 515-518. [CrossRef]

49. Dey, N.; Bhattacherjee, S. Accumulation of polyphenolic compounds and osmolytes under dehydration stress and their implication in redox regulation in four indigenous aromatic rice cultivars. Rice Sci. 2020, 27, 329-344. [CrossRef]

50. Shan, T.; Wang, Y.; Wang, S.; Xie, Y.; Cui, Z.; Wu, C.; Sun, J.; Wang, J.; Mao, Z. A new p-terphenyl derivative from the insect-derived fungus Aspergillus candidus Bdf-2 and the synergistic effects of terphenyllin. Peer J. 2020, 8, e8221. [CrossRef] [PubMed]

51. Bao, J.; Sun, Y.L.; Zhang, X.Y.; Han, Z.; Gao, H.C.; He, F.; Qian, P.Y.; Qi, S.H. Antifouling and antibacterial polyketides from marine gorgonian coral-associated fungus Penicillium sp. SCSGAF 0023. J. Antibiot. 2013, 66, 219-223. [CrossRef]

52. Munden, J.E.; Butterworth, D.; Hanscomb, G.; Verrall, M.S. Production of chlorflavonin, an antifungal metabolite of Aspergillus candidus. Appl. Microbiol. 1970, 19, 718-720. [CrossRef]

53. Ma, J.; Zhang, X.L.; Wang, Y.; Zheng, J.Y.; Wang, C.Y.; Shao, C.L. Aspergivones A and B, two new flavones isolated from a gorgonian-derived Aspergillus candidus fungus. Nat. Prod. Res. 2017, 31, 32-36. [CrossRef] [PubMed]

54. Watanabe, S.; Hirai, H.; Kato, Y.; Nishida, H.; Saito, T.; Yoshikawa, N.; Kojima, Y. CJ-19,784, a new antifungal agent from a fungus, Acanthostigmella sp. J. Antibiot. 2001, 54, 1031-1035. [CrossRef]

55. Pan, Y.; Zheng, W.; Yang, S. Chemical and activity investigation on metabolites produced by an endophytic fungi Psathyrella candolleana from the seed of Ginkgo biloba. Nat. Prod. Res. 2019, 133, 1-4. [CrossRef]

56. Nakayama, O.; Yagi, M.; Tanaka, M.; Kiyoto, S.; Uchida, I.; Hashimoto, M.; Okuhara, M.; Kohsaka, M. WS-7528, a new isoflavanone with estrogen activity isolated from Streptomyces sp. No. 7528. Taxonomy, fermentation, isolation, physico-chemical properties and biological activities. J. Antibiot. 1990, 43, 1394-1402. [CrossRef] 
57. El-Gendy, M.M.; Shaaban, M.; El-Bondkly, A.M.; Shaaban, K.A. Bioactive benzopyrone derivatives from new recombinant fusant of marine Streptomyces. Appl. Biochem. Biotechnol. 2008, 150, 85-96. [CrossRef] [PubMed]

58. Jiang, Z.D.; Jensen, P.R.; Fenical, W. Actinoflavoside, a novel flavonoid-like glycoside produced by a marine bacterium of the genus Streptomyces. Tetrahedron Lett. 1997, 38, 5065-5068. [CrossRef]

59. Balachandran, C.; Sangeetha, B.; Duraipandiyan, V.; Raj, M.K.; Ignacimuthu, S.; Al-Dhabi, N.A.; Balakrishna, K.; Parthasarathy, K.; Arulmozhi, N.M.; Arasu, M.V. A flavonoid isolated from Streptomyces sp. (ERINLG-4) induces apoptosis in human lung cancer A549 cells through p53 and cytochrome c release caspase dependant pathway. Chem. Biol. Interact. 2014, 224, 24-35. [CrossRef]

60. Cao, D.D.; Do, T.Q.; Doan Thi Mai, H.; Vu Thi, Q.; Nguyen, M.A.; Le Thi, H.M.; Tran, D.T.; Chau, V.M.; Cong Thung, D.; Pham, V.C. Antimicrobial lavandulylated flavonoids from a sponge-derived actinomycete. Nat. Prod. Res. 2020, 34, 413-420. [CrossRef] [PubMed]

61. Cao, D.D.; Trinh, T.T.V.; Mai, H.D.T.; Vu, V.N.; Le, H.M.; Thi, Q.V.; Nguyen, M.A.; Duong, T.T.; Tran, D.T.; Chau, V.M.; et al. Antimicrobial lavandulylated flavonoids from a sponge-derived Streptomyces sp. G248 in east vietnam sea. Mar. Drugs 2019, 17, 529. [CrossRef]

62. Da Silva, B.F.; Rodrigues-Fo, E. Production of a benzylated flavonoid from 5,7, $3^{\prime}, 4^{\prime}, 5^{\prime}$-pentamethoxyflavanone by Penicillium griseoroseum. J. Mol. Catal. B Enzym. 2010, 67, 184-188. [CrossRef]

63. Anyanwutaku, I.O.; Zirbes, E.; Rosazza, J.P.N. Isoflavonoids from Streptomycetes: Origins of genistein, 8-chlorogenistein, and 6,8-dichlorogenistein. J. Nat. Prod. 1992, 55, 1498-1504. [CrossRef]

64. Das, S.; Rosazza, J.P.N. Microbial and enzymatic transformations of flavonoids. J. Nat. Prod. 2006, 69, 499-508. [CrossRef] [PubMed]

65. Hideo, E.; Ryoko, W.; Hiromichi, O.; Shunro, K.; Toshihiko, O. Formation mechanism for potent antioxidative o-dihydroxyisoflavones in soybeans fermented with Aspergillus saitoi. Biosci. Biotechnol. Biochem. 1999, 63, 851-858. [CrossRef]

66. Miyazawa, M.; Ando, H.; Okuno, Y.; Araki, H. Biotransformation of isoflavones by Aspergillus niger, as biocatalyst. J. Mol. Catal. B Enzym. 2004, 27, 91-95. [CrossRef]

67. Miyazawa, M.; Takahashi, K.; Araki, H. Biotransformation of isoflavones by Aspergillus niger as biocatalyst. J. Mol. Catal. B Enzym. 2006, 81, 674-678. [CrossRef]

68. Tahara, S.; Ingham, J.L.; Mizutani, J. Identification of an epoxy-intermediate resulting from the fungal metabolism of a prenylated isoflavone. Phytochemistry 1989, 28, 2079-2084. [CrossRef]

69. Kostrzewa-Susłow, E.; Dmochowska-Gładysz, J.; Białońska, A.; Ciunik, Z.; Rymowicz, W. Microbial transformations of flavanone and 6-hydroxyflavanone by Aspergillus niger strains. J. Mol. Catal. B Enzym. 2006, 39, 18-23. [CrossRef]

70. Tahara, S.; Nakahara, S.; Ingham, J.L.; Mizutani, J. Fungal metabolites of antifungal isoflavone wighteone. J. Chem. Soc. Jpn. 1985, 59, 1039-1044. [CrossRef]

71. Ye, H.; Yuan, S.; Cong, X.d. Biotransformation of puerarin into 3'-hydroxypuerarin by Trichoderma harzianum NJ01. Enzym. Microb. Technol. 2007, 40, 594-597. [CrossRef]

72. Mohamed, A.E.H.H.; Khalafallah, A.K.; Yousof, A.H. Biotransformation of glabratephrin, a rare type of isoprenylated flavonoids, by Aspergillus niger. Z. Naturforsch. C 2008, 63, 561-564. [CrossRef] [PubMed]

73. Sasaki, K.; Tsurumaru, Y.; Yazaki, K. Prenylation of flavonoids by biotransformation of yeast expressing plant membrane-bound prenyltransferase SfN8DT-1. Biosci. Biotechnol. Biochem. 2009, 73, 759-761. [CrossRef] [PubMed]

74. Kostrzewa-Susłowa, E.; Dmochowska-Gładysza, J.; Janeczkoa, T.; Srodab, K.; Michalakb, K.; Palkob, A. Microbial transformations of 6- and 7-methoxyflavones in Aspergillus niger and Penicillium chermesinum cultures. Z. Naturforsch. C J. Biosci. 2012, 67, 411-417. [CrossRef]

75. Kostrzewa-Suslow, E.; Janeczko, T. Microbial transformations of 7-hydroxyflavanone. Sci. World J. 2012, 2012, 254929. [CrossRef]

76. Herath, W.; Ferreira, D.; Khan, S.I.; Khan, I.A. Identification and biological activity of microbial metabolites of xanthohumol. Chem. Pharm. Bull. 2003, 51, 1237-1240. [CrossRef] [PubMed]

77. Tian, Y.; Amand, S.; Buisson, D.; Kunz, C.; Hachette, F.; Dupont, J.; Nay, B.; Prado, S. The fungal leaf endophyte Paraconiothyrium variabile specifically metabolizes the host-plant metabolome for its own benefit. Phytochemistry 2014, 108, 95-101. [CrossRef] [PubMed]

78. Lee, J.H.; Oh, E.T.; Chun, S.C.; Keum, Y.S. Biotransformation of isoflavones by Aspergillus niger and Cunninghamella elegans. J. Korean Soc. Appl. Biol. Chem. 2014, 57, 523-527. [CrossRef] 
79. Popłoński, J.; Sordon, S.; Tronina, T.; Bartmańska, A.; Huszcza, E. Fungal metabolism of naphthoflavones. J. Mol. Catal. B Enzym. 2015, 117, 1-6. [CrossRef]

80. Ma, S.; Zheng, C.; Feng, L.; Huang, Y.W.; Wang, R.; Huang, C.; Li, Y.M.; Guo, F.J. Microbial transformation of prenylflavonoids from Psoralea corylifolia by using Cunninghamella blakesleeana and C. elegans. J. Mol. Catal. B Enzym. 2015, 118, 8-15. [CrossRef]

81. Dymarska, M.; Grzeszczuk, J.; Urbaniak, M.; Janeczko, T.; Plaskowska, E.; Stepien, L.; Kostrzewa-Suslow, E. Glycosylation of 6-methylflavone by the strain Isaria fumosorosea KCH J2. PLoS ONE 2017, 12, e0184885. [CrossRef]

82. Dymarska, M.; Janeczko, T.; Kostrzewa-Suslow, E. Biotransformations of flavones and an Isoflavone (Daidzein) in cultures of entomopathogenic filamentous fungi. Molecules 2018, 23, 1356. [CrossRef]

83. Dymarska, M.; Janeczko, T.; Kostrzewa-Suslow, E. Glycosylation of methoxylated flavonoids in the cultures of isaria fumosorosea $\mathrm{KCH}$ J2. Molecules 2018, 23, 2578. [CrossRef]

84. Dymarska, M.; Janeczko, T.; Kostrzewa-Suslow, E. Glycosylation of 3-hydroxyflavone, 3-methoxyflavone, quercetin and baicalein in fungal cultures of the genus Isaria. Molecules 2018, 23, 2477. [CrossRef] [PubMed]

85. Dou, F.; Wang, Z.; Li, G.; Dun, B. Microbial transformation of flavonoids by Isaria fumosorosea ACCC 37814. Molecules 2019, 24, 1028. [CrossRef]

86. Harwoko, H.; Hartmann, R.; Daletos, G.; Ancheeva, E.; Frank, M.; Liu, Z.; Proksch, P. Biotransformation of host plant flavonoids by the fungal endophyte Epicoccum nigrum. ChemistrySelect 2019, 4, 13054-13057. [CrossRef]

87. Luzny, M.; Tronina, T.; Kozlowska, E.; Dymarska, M.; Poplonski, J.; Lyczko, J.; Kostrzewa-Suslow, E.; Janeczko, T. Biotransformation of methoxyflavones by selected entomopathogenic filamentous fungi. Int. J. Mol. Sci. 2020, 21, 6121. [CrossRef]

88. Alarcón, J.; Alderete, J.; Escobar, C.; Araya, R.; Cespedes, C.L. Aspergillus niger catalyzes the synthesis of flavonoids from chalcones. Biocatal. Biotransformation 2013, 31, 160-167. [CrossRef]

89. Chun, H.K.; Ohnishi, Y.; Shindo, K.; Misawa, N.; Furukawa, K.; Horinouchi, S. Biotransformation of flavone and flavanone by Streptomyces lividans cells carrying shuffled biphenyl dioxygenase genes. J. Mol. Catal. B Enzym. 2003, 21, 113-121. [CrossRef]

90. Kumano, T.; Richard, S.B.; Noel, J.P.; Nishiyama, M.; Kuzuyama, T. Chemoenzymatic syntheses of prenylated aromatic small molecules using Streptomyces prenyltransferases with relaxed substrate specificities. Bioorg. Med. Chem. Lett. 2008, 16, 8117-8126. [CrossRef]

91. Hu, S.C.; Hong, K.; Song, Y.C.; Liu, J.Y.; Tan, R.X. Biotransformation of soybean isoflavones by a marine Streptomyces sp. 060524 and cytotoxicity of the products. World J. Microb. Biot. 2008, 25, 115-121. [CrossRef]

92. Marvalin, C.; Azerad, R. Microbial glucuronidation of polyphenols. J. Mol. Catal. B Enzym. 2011, 73, 43-52. [CrossRef]

93. Ma, B.; Zeng, J.; Shao, L.; Zhan, J. Efficient bioconversion of quercetin into a novel glycoside by Streptomyces rimosus subsp. rimosus ATCC 10970. J. Biosci. Bioeng. 2013, 115, 24-26. [CrossRef]

94. Liu, L.; Yao, Q.; Ma, Z.; Ikeda, H.; Fushinobu, S.; Xu, L.H. Hydroxylation of flavanones by cytochrome P450 105D7 from Streptomyces avermitilis. J. Mol. Catal. B Enzym. 2016, 132, 91-97. [CrossRef]

95. Parajuli, P.; Pandey, R.P.; Nguyen, T.H.T.; Dhakal, D.; Sohng, J.K. Substrate Scope of O-Methyltransferase from Streptomyces peucetius for Biosynthesis of Diverse Natural Products Methoxides. Appl. Biochem. Biotechnol. 2018, 184, 1404-1420. [CrossRef] [PubMed]

96. Ibrahim, A.R.; Abulhajj, Y.J. Microbiological transformation of chromone, chromanone, and ring a hydroxyflavones. J. Nat. Prod. 1990, 53, 1471-1478. [CrossRef]

97. Song, M.C.; Kim, E.J.; Kim, E.; Rathwell, K.; Nam, S.J.; Yoon, Y.J. Microbial biosynthesis of medicinally important plant secondary metabolites. Nat. Prod. Rep. 2014, 31, 1497-1509. [CrossRef] [PubMed]

98. Nguyen, C.T.; Dhakal, D.; Pham, V.T.T.; Nguyen, H.T.; Sohng, J.K. Recent advances in strategies for activation and discovery/characterization of cryptic biosynthetic gene clusters in Streptomyces. Microorganisms 2020, 8 , 616. [CrossRef]

99. Lee, Y.; Lee, N.; Hwang, S.; Kim, K.; Kim, W.; Kim, J.; Cho, S.; Palsson, B.O.; Cho, B.K. System-level understanding of gene expression and regulation for engineering secondary metabolite production in Streptomyces. J. Ind. Microbiol. Biotechnol. 2020. [CrossRef]

100. Kaneko, M.; Ohnishi, Y.; Horinouchi, S. Cinnamate: Coenzyme A ligase from the filamentous bacterium Streptomyces coelicolor A3(2). J. Bacteriol. 2003, 185, 20-27. [CrossRef] 
101. Horinouchi, S. Mining and polishing of the treasure trove in the bacterial genus Streptomyces. Biosci. Biotechnol. Biochem. 2007, 71, 283-299. [CrossRef]

102. Hwang, E.I.; Kaneko, M.; Ohnishi, Y.; Horinouchi, S. Production of plant-specific flavanones by Escherichia coli containing an artificial gene cluster. Appl. Environ. Microbiol. 2003, 69, 2699-2706. [CrossRef]

103. Kim, B.G.; Jung, B.R.; Lee, Y.; Hur, H.G.; Lim, Y.; Ahn, J.H. Regiospecific flavonoid 7-O-methylation with Streptomyces avermitiliso methyltransferase expressed in Escherichia coli. J. Agric. Food Chem. 2006, 54, 823-828. [CrossRef]

104. Koirala, N.; Pandey, R.P.; Parajuli, P.; Jung, H.J.; Sohng, J.K. Methylation and subsequent glycosylation of 7,8-dihydroxyflavone. J. Biotechnol. 2014, 184, 128-137. [CrossRef] [PubMed]

105. Darsandhari, S.; Dhakal, D.; Shrestha, B.; Parajuli, P.; Seo, J.H.; Kim, T.S.; Sohng, J.K. Characterization of regioselective flavonoid O-methyltransferase from the Streptomyces sp. KCTC 0041BP. Enzym. Microb. Technol. 2018, 113, 29-36. [CrossRef] [PubMed]

106. Choi, S.H.; Ryu, M.; Yoon, Y.J.; Kim, D.M.; Lee, E.Y. Glycosylation of various flavonoids by recombinant oleandomycin glycosyltransferase from Streptomyces antibioticus in batch and repeated batch modes. Biotechnol. Lett. 2012, 34, 499-505. [CrossRef]

107. Park, S.R.; Yoon, J.A.; Paik, J.H.; Park, J.W.; Jung, W.S.; Ban, Y.H.; Kim, E.J.; Yoo, Y.J.; Han, A.R.; Yoon, Y.J. Engineering of plant-specific phenylpropanoids biosynthesis in Streptomyces venezuelae. J. Biotechnol. 2009, 141, 181-188. [CrossRef] [PubMed]

108. Marin, L.; Gutierrez-Del-Rio, I.; Entrialgo-Cadierno, R.; Villar, C.J.; Lombo, F. De novo biosynthesis of myricetin, kaempferol and quercetin in Streptomyces albus and Streptomyces coelicolor. PLoS ONE 2018, 13, e0207278. [CrossRef]

109. Koeduka, T.; Shitan, N.; Kumano, T.; Sasaki, K.; Sugiyama, A.; Linley, P.; Kawasaki, T.; Ezura, H.; Kuzuyama, T.; Yazaki, K. Production of prenylated flavonoids in tomato fruits expressing a prenyltransferase gene from Streptomyces coelicolor A3(2). Plant Biol. 2011, 13, 411-415. [CrossRef]

110. Alvarez-Alvarez, R.; Botas, A.; Albillos, S.M.; Rumbero, A.; Martin, J.F.; Liras, P. Molecular genetics of naringenin biosynthesis, a typical plant secondary metabolite produced by Streptomyces clavuligerus. Microb. Cell Fact. 2015, 14, 178. [CrossRef]

111. Merkens, H.; Sielker, S.; Rose, K.; Fetzner, S. A new monocupin quercetinase of Streptomyces sp. FLA: Identification and heterologous expression of the queD gene and activity of the recombinant enzyme towards different flavonols. Arch. Microbiol. 2007, 187, 475-487. [CrossRef]

112. Smith, R.V.; Rosazza, J.P. Microbial models of mammalian metabolism. J. Pharm. Sci. 1975, 64, 1737-1759. [CrossRef]

113. Davis, P.J.; Yang, S.K.; Smith, R.V. Microbial models of mammalian metabolism: Stereospecificity of ketone reduction with pentoxifylline. Xenobiotica 1985, 15, 1001-1010. [CrossRef]

114. Ibrahim, A.R.S.; Abul-hajj, Y.J. Microbiological transformation of flavone and isoflavone. Xenobiotica 1990, 20, 363-373. [CrossRef]

Publisher's Note: MDPI stays neutral with regard to jurisdictional claims in published maps and institutional affiliations.

(C) 2020 by the authors. Licensee MDPI, Basel, Switzerland. This article is an open access article distributed under the terms and conditions of the Creative Commons Attribution (CC BY) license (http://creativecommons.org/licenses/by/4.0/). 